\title{
WEALTH AS A SIGNAL IN THE SEARCH MODEL OF MONEY
}

\author{
Tsunao Okumura ${ }^{1}$ \\ International Graduate School of Social Sciences, \\ Yokohama National University, Japan, and \\ Department of Economics, \\ Northwestern University, U.S.A.
}

June 2005

An abridged version of this paper will appear at the International Economic Review.

\begin{abstract}
This paper investigates the possibility that wealth (holdings of money) serves as a signal of ability to produce high quality products for agents who cannot directly observe the quality of the products. A producer's wealth may advertise past success in selling products to agents who knew the producer's ability and thus signal its ability. This analysis shows that such signaling effects may arise in equilibrium and may lead to more unequal distributions of wealth and lower welfare than would otherwise arise.

JEL Classification: E40, D82, D83, D31

Keywords: Random matching, Money holdings, Signaling, Distribution of wealth, Welfare, Divisible money, Product quality.

\footnotetext{
${ }^{1}$ This paper is a revised chapter of my dissertation at Northwestern University. Sincere gratitude is extended to Gadi Barlevy, Kiminori Matsuyama, Dale Mortensen, Lars Stole, Alberto Trejos and Asher Wolinsky for their guidance, encouragement and helpful comments. I am grateful to the editor Randall Wright and three anonymous referees for their very helpful suggestions. I am also grateful to Boyan Jovanovic, Nobuhiro Kiyotaki, Yoshiyasu Ono, Craig Parsons, Makoto Shimoji, Emiko Usui, Randal Watson, Ruilin Zhou and seminar participants at the 2000 Society for Economic Dynamics Conference, the 2000 Midwest Macroeconomics Conference, Northwestern University, International Economics and Finance Society Japan, the Kansai Macroworkshop, University of Tokyo, Tsukuba University and Yokohama National University for their helpful comments. Financial support from the Japan Society for the Promotion of Science and the Japanese Bankers Association are also greatly appreciated. Please address correspondence to: T. Okumura, International Graduate School of Social Sciences, Yokohama National University, 79-4 Tokiwadai Hodogayaku, Yokohama 2408501, Japan. Email: okumura@ynu.ac.jp.
} 


\section{INTRODUCTION}

This paper investigates the possibility that wealth (holdings of money) serves as a signal of a producer's ability when not all agents can directly observe that ability. The analysis shows that such signaling effects are theoretically plausible (i.e., may arise in equilibrium) and may lead to more unequal distributions of wealth and lower welfare than would otherwise arise.

The basic framework employed here is a combination of a search-theoretic model of the exchange process and Spence's (1973) signaling model. Kiyotaki and Wright (1989, 1991, 1993) presented a search-theoretic equilibrium model of the exchange process, in which the "double coincidence of wants" problem generates a role for money as a medium of exchange. Williamson and Wright (1994) extended Kiyotaki and Wright's model to a model of production and exchange with uncertainty concerning the quality of commodities. Sellers choose the quality of their commodities and buyers cannot perfectly observe the quality of a trading partner's commodity. In this case, private information may lead to lemons and moral hazard problems: agents producing low-quality commodities. They show that introduction of money ameliorates frictions caused by lemons and moral hazard problems.

This paper extends Williamson and Wright's (1994) model to investigate the signaling effect of the holding of money on the distribution of wealth and welfare. To this end, we assume that (1) an agent cannot perfectly observe a trading partner's innate ability to produce high-quality commodity and (2) an agent can hold multiple units of money.

Asymmetric information about product quality is used in the model below, via a classification of agents into innate high-ability and low-ability producers. In any single meeting an agent is either a buyer or a seller. As buyers, they naturally prefer to purchase higher quality products (from high-ability producers), but they are not certain of being able to identify the high-ability sellers when they meet them. However, they can observe a seller's money holdings, and in some situations may interpret these holdings as a signal of previous success in selling high-quality products. To permit such signaling effects, this paper relaxes the assumption of single-unit money holdings to admit the accumulation of multiple units of money. 
The signaling role for wealth holdings is introduced by randomly allowing some buyers to receive private information on the quality of sellers' products. These buyers will then definitely trade when they meet high-ability sellers, and such sellers therefore tend to accumulate money. At an equilibrium, buyers without private information predict that wealthy agents are more likely to be high-ability agents, and therefore are more willing to trade with them. Conversely, they are less willing to trade with poor agents. Thus the accumulation of money is used as a signal. That is, buyers who do not know the quality of products believe that wealthy people became rich because they could produce high-quality products, whereas sellers covet wealth in order to be regarded as able producers, making their products easy to sell to buyers who do not know their quality. As a result of a wealth-signaling effect, agents save more (or spend less) money to signal their ability as producers, and those with wealth increase sales more easily than those without. Thus, the distribution of wealth becomes more unequal than in an economy where agents do not judge producers' quality by their wealth. Wealth signaling has the following effects on welfare. The positive effect helps agents better identify product quality and hence ameliorate the private information problem. The negative effect, however, reduces opportunities for trade for the following reasons. Firstly, agents hold on to wealth that might otherwise be used for purchases. Secondly, both high- and low- ability agents have more difficulty in selling their products when they do not have wealth. Thirdly, high-ability agents concentrate in the wealthy (or leisure) class the members of which are only buyers and hence, the production of high-quality commodities decreases. A numerical comparison of welfare levels of high- and low-ability agents shows that this unfavorable effect on welfare is dominant for both types.

The assumption that buyers observe sellers' money holdings reflects the fact that in the real world, the profits of trade are often converted into shows of disposal wealth such as savings, estates and works of art.

Many examples can be suggested. Consumers may trust the quality of the products of "large" companies, such as those which run many factories and own splendid buildings. It may also be easier for "large" companies to raise funds than "small" companies. A lawyer, a doctor, a dealer 
and a private detective often decorate their offices with expensive paintings, wear luxury suits and drive luxury cars. These businesses could appeal to new customers by showing off their wealth. When one visits an area for the first time, one often does not want to eat out at a shabby restaurant.

Trejos and Wright (1995), Green and Zhou (1998), Molico (1998), Camera and Corbae (1999), Taber and Wallace (1999), Zhou (1999), Berentsen (2000, 2002) and Berentsen et al. (2002) allowed agents to accumulate money by relaxing the one-unit constraint on money holdings and/or the fixed price assumption, both of which are assumed in Kiyotaki and Wright (1989, 1991, 1993). In Section 2 and 3, this paper presents a model in which agents accumulate money up to the fixed bound and trade at the fixed price to investigate equilibria wherein agents' money holdings signal their type. Section 4 extends the model by allowing agents to trade at an endogenous price and hold an arbitrary amount of money (referring to Zhou (1999)) to analyze the signaling single-price equilibria and establish that the main conclusions do not change in the equilibria.

The rest of the paper is organized as follows. Section 2 presents the model. Section 3 analyzes the equilibria where the accumulation of money is used as a signal. Section 4 investigates the signaling equilibria where price and upper bounds on money holdings are endogenously determined. Section 5 concludes the paper.

\section{THE MODEL}

Time is continuous and infinite. There is a continuum of measure one of infinitely-lived agents in the economy. Agents (and commodities) are categorized into types according to a twodimensional classification: each agent is associated with an ability (or quality) level (high $K=H$ or low $K=L$ ), and the single variety of the commodity that they produce (indexed by $j=$ $1, \ldots, J)$. In total there are $2 J$ types of agents (or commodities) indexed $j$ - $K$. The proportion $\mu$ of high-ability producers of a variety is the same for all $j=1, \ldots, J$. The population of producers 
of any variety $j$ has measure $1 / J$. Thus abilities and varieties are independently distributed in the population. Each agent, whether of high ability or low, can produce one unit of their variety at a positive per-unit utility cost of $\gamma$ instantaneously at any time (even when she holds money). However type $H$ agents produce the variety at a high level of quality, whereas type $L$ agents can only make a low-quality version.

In addition to commodities, there is money; all commodities and money are indivisible and freely disposable. Money is perfectly durable and storable at zero cost, and thus can be accumulated, whereas commodities are nonstorable. Each agent only gets positive utility from the consumption of one unit of one specific variety and quality combination. In particular, a variety- $j$ producer derives utility $u>0$ from consumption of a high-quality unit of variety $j+1$, and zero utility from the consumption of any other kind of commodity, or money ${ }^{2}$. Money cannot be produced by any private agent. At the beginning of the initial period, a measure $\bar{M}$ of money is allocated at random in the economy.

In each period, agents meet pairwise and at random according to a Poisson process with constant arrival rate $\beta>0$. Denote $\alpha \equiv \beta / J$. When two agents meet, they decide whether or not to trade. In any meeting, an agent can identify which variety of the commodity a trading partner produces. Since there is no barter, a type $j$ agent becomes a buyer when she has money and meets a type $j+1$ agent and a seller when she meets a type $j-1$ agent.

In any meeting, a buyer can identify how much money a trading partner (a seller) has. She recognizes the quality of the commodity that a trading partner produces with probability $\theta$. This probability is independent across sellers and meetings. A seller does not know if a buyer recognizes the quality of her commodity, while a buyer does not know anything about a seller's history in meetings. In a meeting, a buyer inspects the quality of the seller's commodity and buyer and seller then simultaneously announce strategies from \{buy, no trade $\}$ and \{sell, no trade\} respectively. Trade only takes place if one party announces 'buy' and the other announces 'sell'. Trade entails a swap of a unit of a commodity for a unit of money. (The price

\footnotetext{
${ }^{2}$ The preferred consumption variety for variety- $J$ agents is a high-quality unit of variety 1.
} 
of the commodity is constant.) Production, trading and consumption are on the spot due to nonstorable commodities. Money cannot be exchanged for money.

Agents choose production, consumption and trading strategies in order to maximize the expected utility of consumption, taking as given the other agent's strategy. We look for Nash equilibria. We restrict attention to equilibria that are symmetric, stationary and active (some trade takes place). We consider equilibria wherein a buyer who recognizes the quality of the seller's commodity wants to trade only with the high-ability sellers. ${ }^{3}$

Let $V_{K}(m)$ denote the value function for the agent who is type- $K(K=H, L)$ with $m$ units of money. Let $\Sigma_{K}(m, \widetilde{m})$ denote the probability that a random type- $K$ uninformed buyer with $m$ units of money announces 'buy' in meetings with a seller with $\widetilde{m}$ units of money. Let $\Phi_{K}(m)$ denote the probability that a random type- $K$ seller with $m$ units of money announces 'sell' in meetings with a buyer. Let $\sigma_{K}(m, \widetilde{m})$ denote the best response of a representative type- $K$ uninformed buyer with $m$ units of money in meetings with a seller with $\widetilde{m}$ units of money, and $\phi_{K}(m)$ denote the best response of a representative type- $K$ seller with $m$ units of money. $P_{m}^{K}$ is the measure of type- $K$ agents with $m$ units of money, $M_{K}$ is the maximum amount of money a type- $K$ agent holds and $r$ is the rate of time preference. The Bellman equations are given by

For $K=H, L$ :

\footnotetext{
${ }^{3}$ Only one example in subsection 3.6 considers an equilibria wherein some informed buyers do not buy commodities from the high-ability sellers.
} 
For $m=1,2, \ldots, M_{K}-1$,

$$
\begin{aligned}
& r V_{K}(m) \\
& =\alpha\left((1-\theta) \sum_{\widetilde{m}=0}^{M-1} P_{\widetilde{m}} \max _{\sigma_{K}(m, \widetilde{m})} \sigma_{K}(m, \widetilde{m})\left[\mu_{\tilde{m}} u+V_{K}(m-1)-V_{K}(m)\right] \Phi(\widetilde{m})\right. \\
& +\theta \sum_{\widetilde{m}=0}^{M-1} P_{\tilde{m}}^{H}\left[u+V_{K}(m-1)-V_{K}(m)\right] \Phi_{H}(\widetilde{m}) \\
& +\left\{(1-\theta) \sum_{\widetilde{m}=1}^{M}\left[P_{\tilde{m}}^{H} \Sigma_{H}(\widetilde{m}, m)+P_{\tilde{m}}^{L} \Sigma_{L}(\widetilde{m}, m)\right]+1_{K} \theta \sum_{\widetilde{m}=1}^{M} P_{\widetilde{m}}\right\} \\
& \left.\times \max _{\phi_{K}(m)} \phi_{K}(m)\left[V_{K}(m+1)-\gamma-V_{K}(m)\right]\right)
\end{aligned}
$$

$$
\begin{aligned}
& r V_{K}(0) \\
& =\alpha\left\{(1-\theta) \sum_{\widetilde{m}=1}^{M}\left[P_{\tilde{m}}^{H} \Sigma_{H}(\widetilde{m}, 0)+P_{\widetilde{m}}^{L} \Sigma_{L}(\widetilde{m}, 0)\right]+1_{K} \theta \sum_{\widetilde{m}=1}^{M} P_{\widetilde{m}}\right\} \\
& \times \max _{\phi_{K}(0)} \phi_{K}(0)\left[V_{K}(1)-\gamma-V_{K}(0)\right]
\end{aligned}
$$

$$
\begin{aligned}
& r V_{K}\left(M_{K}\right) \\
& =\alpha\left\{(1-\theta) \sum_{\widetilde{m}=0}^{M-1} P_{\widetilde{m}} \max _{\sigma_{K}\left(M_{K}, \widetilde{m}\right)} \sigma_{K}\left(M_{K}, \widetilde{m}\right)\left[\mu_{\widetilde{m}} u+V_{K}\left(M_{K}-1\right)-V_{K}\left(M_{K}\right)\right] \Phi(\widetilde{m})\right. \\
& \left.+\theta \sum_{\widetilde{m}=0}^{M-1} P_{\widetilde{m}}^{H}\left[u+V_{K}\left(M_{K}-1\right)-V_{K}\left(M_{K}\right)\right] \Phi_{H}(\widetilde{m})\right\},
\end{aligned}
$$

where $1_{K}=1$ if $K=H$ and 0 if $K=L, P_{m}=P_{m}^{H}+P_{m}^{L}, \mu_{m}=P_{m}^{H} / P_{m}, \Phi(m)=$ $\mu_{m} \Phi_{H}(m)+\left(1-\mu_{m}\right) \Phi_{L}(m)$ and $M=\max \left(M_{H}, M_{L}\right)$. 
Each equation has the following interpretation. In equation (1), the flow return to an agent who holds $m$ units of money equals the sum of the gains when an agent is a buyer (the first and second terms) and when she is a seller (the third term). The first term is the probability that the agent meets someone with her favorite variety but with unidentified quality, times the gains from a purchase with probability $\sigma_{K}(m, \widetilde{m})$, given a seller's offer. The second term is the probability that the agent meets someone with her favorite variety of known quality, times the gain from the trade, given a seller's offer. The third term is the probability that the agent meets someone who prefers the variety of her commodity, times the probability that the trading partner is willing to buy it, times the gains from trade. The crucial difference between equations for high $(K=H)$ and low $(K=L)$ types is that for the low-type agents $\theta \sum_{\widetilde{m}=1}^{M} P_{\widetilde{m}}$ is not in the third term, since the informed buyer would like to trade only with the high-ability sellers. Equations (2) and (3) represent the value functions at the bounds of $m=0$ and $M_{K}$. When $\theta$ equals unity and there is no low-ability agent, the high-ability agent's value functions are similar to the value functions presented in Camera and Corbae (1999), Zhou (1999) and Berentsen (2000, 2002).

In any symmetric stationary equilibrium, the flow out of each state equals the flow into this state, $\sigma_{K}(m, \widetilde{m})=\Sigma_{K}(m, \widetilde{m})$ and $\phi_{K}(m)=\Phi_{K}(m)$, thus:

For $m=1,2, \ldots, M_{K}-1$,

$$
\begin{aligned}
& P_{m+1}^{K}\left[(1-\theta) \sum_{\widetilde{m}=0}^{M-1} P_{\widetilde{m}} \Sigma_{K}(m+1, \widetilde{m}) \Phi(\widetilde{m})+\theta \sum_{\widetilde{m}=0}^{M-1} P_{\widetilde{m}}^{H} \Phi_{H}(\widetilde{m})\right] \\
& +P_{m-1}^{K} \Phi_{K}(m-1)\left\{(1-\theta) \sum_{\widetilde{m}=1}^{M}\left[P_{\widetilde{m}}^{H} \Sigma_{H}(\widetilde{m}, m-1)+P_{\widetilde{m}}^{L} \Sigma_{L}(\widetilde{m}, m-1)\right]+1_{K} \theta \sum_{\widetilde{m}=1}^{M} P_{\widetilde{m}}\right\} \\
& =P_{m}^{K}\left[(1-\theta) \sum_{\widetilde{m}=0}^{M-1} P_{\widetilde{m}} \Sigma_{K}(m, \widetilde{m}) \Phi(\widetilde{m})+\theta \sum_{\widetilde{m}=0}^{M-1} P_{\widetilde{m}}^{H} \Phi_{H}(\widetilde{m})\right] \\
& +P_{m}^{K} \Phi_{K}(m)\left\{(1-\theta) \sum_{\widetilde{m}=1}^{M}\left[P_{\tilde{m}}^{H} \Sigma_{H}(\widetilde{m}, m)+P_{\widetilde{m}}^{L} \Sigma_{L}(\widetilde{m}, m)\right]+1_{K} \theta \sum_{\widetilde{m}=1}^{M} P_{\widetilde{m}}\right\}
\end{aligned}
$$




$$
\begin{aligned}
& P_{1}^{K}\left[(1-\theta) \sum_{\widetilde{m}=0}^{M-1} P_{\widetilde{m}} \Sigma_{K}(1, \widetilde{m}) \Phi(\widetilde{m})+\theta \sum_{\widetilde{m}=0}^{M-1} P_{\widetilde{m}}^{H} \Phi_{H}(\widetilde{m})\right] \\
& =P_{0}^{K} \Phi_{K}(0)\left\{(1-\theta) \sum_{\widetilde{m}=1}^{M}\left[P_{\widetilde{m}}^{H} \Sigma_{H}(\widetilde{m}, 0)+P_{\widetilde{m}}^{L} \Sigma_{L}(\widetilde{m}, 0)\right]+1_{K} \theta \sum_{\widetilde{m}=1}^{M} P_{\widetilde{m}}\right\}
\end{aligned}
$$

$$
\begin{aligned}
& P_{M_{K}-1}^{K} \Phi_{K}\left(M_{K}-1\right) \\
& \times\left\{(1-\theta) \sum_{\widetilde{m}=1}^{M}\left[P_{\widetilde{m}}^{H} \Sigma_{H}\left(\widetilde{m}, M_{K}-1\right)+P_{\widetilde{m}}^{L} \Sigma_{L}\left(\widetilde{m}, M_{K}-1\right)\right]+1_{K} \theta \sum_{\widetilde{m}=1}^{M} P_{\widetilde{m}}\right\} \\
& =P_{M_{K}}^{K}\left[(1-\theta) \sum_{\widetilde{m}=0}^{M-1} P_{\widetilde{m}} \Sigma_{K}\left(M_{K}, \widetilde{m}\right) \Phi(\widetilde{m})+\theta \sum_{\widetilde{m}=0}^{M-1} P_{\widetilde{m}}^{H} \Phi_{H}(\widetilde{m})\right] \\
& \sum_{m=0}^{M} P_{m}^{H}=\mu, \sum_{m=0}^{M} P_{m}^{L}=1-\mu, \sum_{m=0}^{M} P_{m} m=\bar{M}, P_{m}^{H} \geq 0, P_{m}^{L} \geq 0
\end{aligned}
$$

The left hand side and the right hand side of equation (4) show the flow into and out of type- $K$ agents with $m$ units of money, respectively. Equations (5) and (6) describe the boundary conditions. Equation (7) denotes that the nominal money stock is the sum of money held by all agents. Equations (1)-(7) are the model.

\section{WEALTH-SIGNALING EQUILIBRIA}

This section shows examples of the equilibria where the accumulation of money is used as a signal of ability as a producer, which we, hereafter, refer to as wealth-signaling equilibria. To focus on this objective, in this section we assume that agents cannot hold more than two units of money and study the equilibria wherein both high- and low- type agents act symmetrically, except for in the last example. We investigate two examples of the wealth-signaling equilibria 
and one of the non-wealth-signaling equilibria. Subsequently, we compare their distributions of money and their welfare levels. Finally, we study two other examples of the wealth-signaling equilibria: active signaling and separating equilibria. In numerical analysis, we assume $\mu=1 / 2$ for simplicity.

\subsection{Example 1: Poor Buyers Don't Buy from Poor Sellers. Rich Do.}

This subsection considers the example of a wealth-signaling equilibrium where the uninformed buyers with one unit of money buy only from the sellers with one unit of money (and do not buy from the sellers without money). The uninformed buyers with two units of money buy from either sellers with one unit of money or no money. An uninformed buyer with one unit of money uses a seller's money holdings as a signal of the seller's quality. The sellers announce 'sell' in any meeting. ${ }^{4}$ When $\theta$ is unity so that there is no private information, this equilibrium is related to the single-price equilibria studied in Camera and Corbae (1999), Zhou (1999) and Berentsen $(2000,2002)$.

For this equilibrium to be a Nash equilibrium, i.e., the conditions for the strategy to be a best response for a representative agent:

$$
\gamma<V_{K}(2)-V_{K}(1) \leq \mu_{0} u<V_{K}(1)-V_{K}(0) \leq \mu_{1} u .
$$

Figure 1 shows the existence region of this equilibrium when $\bar{M}=0.75$. The parameters affecting the model are $r / \alpha(\geq 0), \gamma / u(\in[0,1]), \theta(\in[0,1])$ and $\bar{M}(\in[0,2])$. All figures and tables in Section 3 are described for $\bar{M}=0.75$, and some $\theta$. However, we also investigated all cases for other $\bar{M}$ and $\theta$, and found that the interpretation of all figures and tables in Section 3 is also applicable for other $\bar{M}$ and $\theta$. The result is available from the author upon request.

$$
<<\text { Insert Figure 1 }>>
$$

Due to the presence of randomly informed buyers, the fraction of high-type agents who hold one unit of money is larger than the fraction of high-type agents who hold no money. An

\footnotetext{
${ }^{4}$ The sellers, who hold either one unit of money or no money, have the same strategy across all examples save for the last one in this section.
} 
increase in the number of informed agents, $\theta$, enlarges the gap between the two proportions. The uninformed buyers therefore are inclined to trust that money is more informative of the quality of the seller. Consequently, as $\theta$ becomes larger, money becomes a more beneficial signal for the uninformed buyers. However, when $\theta$ is close to one, almost all agents with money are high-type and no low-type agents have money. Thus, no uninformed buyers would like to buy a commodity from sellers without money. These two effects imply that the existence region of this equilibrium is largest for middle sizes of $\theta$.

This equilibrium does not exist either in the region where $r / \alpha$ is large and $\theta$ is small or in the region where $r / \alpha$ is small and $\theta$ is large. For the large discount rate $(r)$ and few opportunities of meeting a trading partner $(\alpha)$, the opportunity cost incurred when an uninformed buyer misses an opportunity to trade with a high-ability seller is large, and thus all uninformed buyers are inclined to trade, especially when $\theta$ is small, meaning money holdings are less informative. In contrast, for small $r / \alpha$, the uninformed buyers tend to avoid the risk involved in buying a commodity with uncertain quality, especially when $\theta$ is large. This interpretation is confirmed by comparison with the existence regions of the second example of the wealth-signaling and of the non-wealth-signaling equilibria as shown below.

The distribution of money (or wealth) is shown in Table 1 when $\bar{M}=0.75 .{ }^{5}$ When $\theta$ equals unity so that there is no private information, $\left(P_{1}^{H}\right)^{2}=P_{0}^{H} P_{2}^{H}$. When $\theta$ equals zero so that there is no informed agent, $\left(P_{1}\right)^{2}=P_{0} P_{2}$. Both distributions of money holdings corresponds with those found in Camera and Corbae (1999), Zhou (1999) and Berentsen (2000, 2002). For larger $\theta$, the proportion of high-ability agents is largest amongst the agents with one and two units of money, and smaller amongst agents with no money. We will compare the distributions of money between wealth-signaling and non-wealth-signaling equilibria in section 3.3.

$$
<<\text { Insert Table } 1>>
$$

\footnotetext{
${ }^{5}$ Since the distribution is determined via equations (4)- (7), the parameters are $\theta$ and $\bar{M}$.
} 


\subsection{The Second Example of Wealth-signaling Equilibrium: Neither Rich nor Poor Buy from the Poor.}

This subsection considers another example of a wealth-signaling equilibrium: an uninformed buyer never buys from a seller without money and she buys from a seller with one unit of money (regardless of her own quantity of money). With this strategy, buyers more severely discriminate between rich and poor sellers than with the strategy in the first example.

The conditions for this equilibrium to be a Nash equilibrium are:

$$
\begin{aligned}
& \max \left(\gamma, \mu_{0} u\right)<V_{H}(1)-V_{H}(0) \leq \mu_{1} u, \max \left(\gamma, \mu_{0} u\right)<V_{H}(2)-V_{H}(1) \leq \mu_{1} u, \\
& \max \left(\gamma, \mu_{0} u\right)<V_{L}(1)-V_{L}(0) \leq \mu_{1} u, \text { and } \max \left(\gamma, \mu_{0} u\right)<V_{L}(2)-V_{L}(1) \leq \mu_{1} u .
\end{aligned}
$$

Figure 2 shows the existence region in the parameter space for this equilibrium. The existence region of this equilibrium becomes larger as $\theta$ increases. As $\theta$ increases, money holdings become more informative of the quality of a seller since there are less high-ability agents in the state without money. Existence in this case holds for smaller $r / \alpha$ than in the first example. For the small $r / \alpha$, or a small opportunity cost of missing trade, the buyers avoid the risk involved in buying a commodity with uncertain quality. The interpretation is applicable for other $\bar{M} \in[0,1]$ and $\theta$.

$<<$ Insert Figure $2>>$

The population at the equilibrium satisfies $\left(P_{1}^{H}\right)^{2}=\theta P_{0}^{H} P_{2}^{H}, P_{1}^{H}+2 P_{2}^{H}=\bar{M}, \sum_{m=0}^{2} P_{m}^{H}=$ $\mu, P_{0}^{L}=1-\mu, P_{1}^{L}=P_{2}^{L}=0$. When $\theta$ equals unity, $\left(P_{1}^{H}\right)^{2}=P_{0}^{H} P_{2}^{H}$, which corresponds with the distributions of money found in Camera and Corbae (1999), Zhou (1999) and Berentsen $(2000,2002)$. The distribution of money is shown in Table 2. Since all type- $L$ agents have no money, the distribution of money is extremely asymmetric across types of agents.

$<<$ Insert Table $2>>$ 


\subsection{Comparison with Non-wealth-signaling Equilibrium}

This subsection considers an example of equilibria where money holdings are not used as a signal: an uninformed buyer buys a commodity regardless of how much money a seller has.

The conditions for this equilibrium to be a Nash equilibrium are:

$$
\gamma<V_{K}(1)-V_{K}(0) \leq \min \left(\mu_{0} u, \mu_{1} u\right) \text { and } \gamma<V_{K}(2)-V_{K}(1) \leq \min \left(\mu_{0} u, \mu_{1} u\right)
$$

Figure 3 shows the existence region of this equilibrium. As $\theta$ decreases, the existence region becomes larger since money holdings are less informative of the quality of the sellers. However, when $\theta$ is large, existence requires a large value for $r / \alpha$ and low $\gamma / u$, since when the net discount rate is large and the production cost is small, the buyers take the risk involved in buying a commodity with uncertain quality.

$<<$ Insert Figure $3>>$

Table 3 shows the distribution of money in the equilibrium. When $\theta$ equals unity, $\left(P_{1}^{H}\right)^{2}=$ $P_{0}^{H} P_{2}^{H}$. When $\theta$ equals zero, $\left(P_{1}\right)^{2}=P_{0} P_{2}$. Both distributions of money holdings correspond with those in example 1 of the wealth-signaling equilibrium and those found in Camera and Corbae (1999), Zhou (1999) and Berentsen (2000, 2002). By comparing the distribution of money with those in the wealth-signaling equilibria of examples 1 and 2 , the concentration of people in the middle class of wealth is found to be greatest in the non-wealth-signaling equilibrium, followed by the signaling equilibrium of example 1, and then that of example 2. The Gini coefficients of the wealth distributions indicate that money is the most equally distributed in the non-signaling equilibrium; example 2 leads to the most unequal distribution and example 1 the second most. For other $\theta$ and $\bar{M}$, the evidence shows that the wealth-signaling equilibrium of example 2 leads to the most unequal distribution of wealth, followed by example 1, with the non-wealth-signaling equilibrium being the most equal. The difference in the degrees of inequality in wealth between the first wealth-signaling and the non-wealth-signaling examples is the largest for middle sizes of $\theta$ and decreases to zero as $\theta$ approaches zero or unity. The difference between the second signaling and the non-signaling examples is the largest in the case of no informed agents $(\theta=0)$ and decreases as $\theta$ increases. 
$<<$ Insert Table $3>>$

\subsection{Multiple Equilibria}

Equilibria co-exist on the regions of the parameter space shared between the examples. Given the distribution of money, the conditions for existence of the equilibria in the examples are mutually exclusive. However, equilibria co-exist because of the following "feedback" effect. Money is more concentrated amongst high-ability agents when money holdings are used as a signal than when money holdings are not used as a signal. Thus, the incentive for the uninformed buyers to consider the sellers' money holdings as a signal is higher when money holdings serve as a signal. Therefore, there exist regions where the wealth-signaling strategy is a best response when money holdings serve as a signal, while the non-wealth-signaling strategy is a best response when money holdings do not serve as a signal.

It is worth emphasizing that regions exist that support a wealth-signaling equilibria and that do not support any non-wealth-signaling equilibria.

\subsection{Welfare}

In this section, we discuss some welfare implications. Type- $K$ 's welfare (or the expected discounted utility of type- $K$ ) is measured by $W_{K}=\sum_{m=0}^{M_{K}} P_{m}^{K} V_{K}(m)$ for $K=H, L$. Figure 4 compares the type- $H$ 's welfare levels, $W_{H}$ for the wealth-signaling equilibria of examples 1 and 2 and the non-wealth-signaling equilibrium. ${ }^{6}$ As the utility level decreases or the production cost increases, the type- $H$ 's welfare in the wealth-signaling equilibrium surpasses that in the non-wealth-signaling equilibrium. As the informed agent's population $(\theta)$ increases and/or the money supply $(\bar{M})$ decreases, the region wherein the type- $H$ 's welfare in the signaling equilibrium is higher than in the non-signaling equilibrium expands. In the co-existence region of the wealth-signaling and non-wealth-signaling equilibria, the type- $H$ 's welfare level is lower for the

\footnotetext{
${ }^{6}$ The results for other $\bar{M}, \theta$ and $r / \alpha$ are available from the author upon request. The following interpretation is also applicable for them.
} 
signaling equilibrium than for the non-signaling equilibrium, except for the region with small $\bar{M}$ and large $\theta$. The second example leads to the lowest welfare level, except for the region with very small $\theta$ and small $\bar{M}$.

Signaling affects welfare in the following ways. The signaling effect helps buyers better identify product quality and hence helps ameliorate the private information problem. However, there are three negative effects. The first negative effect makes agents hoard wealth, decreasing the opportunity for trade. The second negative effect is that even high-ability agents have more difficulty in selling their products when they do not have wealth. The third negative effect decreases the population of the high-types without money, who are high-quality producers (and increases the population of the high-types with two units of money, who are only buyers). The numerical result is consistent with these signaling effects. As the ratio of the production cost to the utility level increases, the uninformed buyers want to avoid the risk involved in buying a commodity with uncertain quality, and as a result use the wealth-signaling. As the population of the informed agents increases, the positive informational effect of signaling on buyers becomes greater and the high-type sellers have less difficulty in selling their products when they do not have wealth. Due to the distributional effect of signaling, the high-types are more concentrated in the state of having two units of money holdings unless the money supply is small and the informed agent's population is large. The numerical result crucially implies that the positive effect is dominated by the negative effects in the co-existence region, except for the region with small $\bar{M}$ and large $\theta .^{7}$

As for low-type agents, the numerical result shows that the welfare level $\left(W_{L}\right)$ is the highest for the non-wealth-signaling equilibrium, followed by welfare in the first wealth-signaling example. The lowest is the welfare in the second wealth-signaling example (which equals zero). Signaling gives the low-types a strong negative effect.

\footnotetext{
${ }^{7}$ Although the increase in $\theta$ enhances the positive effect of signaling, for the co-existence of the second example and the other equilibria, the increase in $\theta$ involves a decrease in the production cost, significantly weakening the positive effect. There is, thus, some co-existence region wherein the welfare level of the second example is not the lowest for very small $\theta$.
} 


\subsection{Active signaling and separating equilibria}

We study two more examples of the wealth-signaling equilibrium: "active" signaling and separating equilibria. An example of the "active" signaling equilibrium has the strategy whereby knowing that agents are discriminated against based on their money holdings, they stop buying when money holdings are low. That is, every (informed and uninformed) agent with one unit of money does not buy and only sell a commodity. The uninformed buyer buys a commodity only from a seller with one unit of money only when she has two units of money. The conditions for this equilibrium are:

$\max \left(\mu_{0} u, \gamma\right)<V_{K}(2)-V_{K}(1) \leq \mu_{1} u$ and $u<V_{K}(1)-V_{K}(0)$.

At the equilibrium, $P_{0}^{H}=0$ and $P_{1}^{H} / P_{1}^{L}=(1-\theta) P_{2}^{H} / P_{2}^{L}$. $P_{0}^{L}$ is set as some initial value. Existence in this case holds for smaller $\theta$ and $r / \alpha$ than in the first and second examples. The increase in population of the informed buyers increases the opportunity for the high-types to escape from the poor and thus inclines the informed high-type buyers with one unit of money to buy from the high-type sellers. As $r / \alpha$ (the net discount rate) gets larger, the uninformed buyers' gain from current purchase surpasses their future gain from the signal. The numerical comparison of the welfare levels shows that this welfare level is lower than the first wealthsignaling and non-wealth-signaling examples for most of the coexistence region. It demonstrates the negative effect of signaling; agents hoard money for signaling and hence the amount of liquidity available for trading is reduced.

The next example is a separating equilibrium wherein high- and low-types do not act symmetrically. That is, high-type agents save money for signaling whereas low-type agents do not. Specifically, high-type agents have the same strategy as in the first wealth-signaling example; low-type agents with one unit of money, however, do not produce, being buyers who buy only from sellers with one unit of money. Thus, high-type's and low-type's upper bounds on money holdings, $M_{H}$ and $M_{L}$, are two and one units, respectively and $M_{L}$ is endogenously determined. The conditions for this equilibrium are:

$$
\gamma<V_{H}(2)-V_{H}(1) \leq \mu_{0} u<V_{H}(1)-V_{H}(0) \leq \mu_{1} u, \mu_{0} u<V_{L}(1)-V_{L}(0) \leq \mu_{1} u, V_{L}(2)-
$$


$V_{L}(1) \leq \gamma<V_{L}(1)-V_{L}(0){ }^{8}$

Existence in this case holds for smaller $\theta$ than in example 1, in which both types have money of up to two units. The increase in the probability of knowing a trading partner's type increases the value of money holdings of two units, thus raising the low-type's incentive to save money. The numerical comparison of the welfare levels shows that the welfare level for this equilibrium is higher than the wealth-signaling and non-wealth-signaling equilibria when the money supply is small and/or the informed agent's population is small.

\section{Endogenous price and upper bounds on money holdings}

In Section 2 and 3, it is assumed that the price and the upper bounds of money holdings are exogenously fixed. This section removes these restrictions, utilizing Zhou's (1999) framework which allows an agent to trade at an endogenous price and hold an arbitrary amount of money. In contrast to Zhou's (1999) assumption that money holdings are private information, this section assumes that sellers' money holdings are observable, whereas buyers' are not.

Some assumptions made in Section 2 are changed into the following. (1) Money is divisible. (All commodities are still indivisible.) An agent can hold any amount of money. (2) In a meeting, a seller posts an offer price at which she is willing to sell and a buyer must either accept or reject it. Trade takes place if and only if the offer is accepted.

In a meeting, a type- $K$ seller makes an offer $\omega_{K}(K=H, L)$. A type- $K$ informed buyer who meets a type- $H$ seller has a reservation-price $\rho_{K}^{I}$, which is a function of her own money holdings. An informed buyer of either type who meets an $L$-type seller has a zero reservation price, since she derives zero utility from the consumption of low-quality products. A type- $K$ uninformed buyer has a reservation-price $\rho_{K}^{U}(\eta, \widetilde{\eta})$, which is a function of both her own money holdings $(\eta)$ and her trading partner's money holdings $(\widetilde{\eta})$, since the uninformed buyer inspects the quality of a seller's commodity observing her trading partner's money holdings. The stationary distribution

\footnotetext{
${ }^{8} V_{L}$ (2) for out-of-equilibrium money holdings is calculated according to Section 4.
} 
of money holdings of type- $K$ agents, $G_{K}$, implies that $\Omega_{K y}^{K^{\prime}}, \Pi_{K y}, R^{U}$ and $R^{I}$ distributions are stationary. $\Omega_{K y}^{K^{\prime}}$ is the distribution of type- $K^{\prime}\left(K^{\prime}=H, L\right)$ agents whose offer price is not higher than the reservation prices of type- $K$ uninformed trading partners with money holdings of $y$. $\Pi_{K y}$ is the distribution of type- $H$ agents whose offer price is not higher than the reservation prices of type- $K$ informed trading partners with money holdings of $y \cdot R^{U}$ is the distribution of the uninformed buyer's reservation price. $R^{I}$ is the distribution of the reservation price of the informed buyer who meets a type- $H$ seller. Specifically,

$$
\begin{aligned}
\Omega_{K y}^{K^{\prime}}(x) & =G_{K^{\prime}}\left\{\eta \mid \omega_{K^{\prime}}(\eta) \leq \rho_{K}^{U}(y, \eta) \wedge \eta \leq x\right\} \\
\Pi_{K y}(x) & =G_{H}\left\{\eta \mid \omega_{H}(\eta) \leq \rho_{K}^{I}(y) \wedge \eta \leq x\right\} \\
R^{U}(x, \widetilde{\eta}) & =G_{H}\left\{\eta \mid \rho_{H}^{U}(\eta, \widetilde{\eta})<x\right\}+G_{L}\left\{\eta \mid \rho_{L}^{U}(\eta, \widetilde{\eta})<x\right\} \\
R^{I}(x) & =G_{H}\left\{\eta \mid \rho_{H}^{I}(\eta)<x\right\}+G_{L}\left\{\eta \mid \rho_{L}^{I}(\eta)<x\right\},
\end{aligned}
$$

Given the reservation price distribution $R^{I}$ and $R^{U}$, the seller posts an offer to maximize her expected discounted net gain from the trade. Then, the buyer checks the offer against her reservation price $\rho_{K}^{U}(\eta, \widetilde{\eta})$ or $\rho_{K}^{I}(\eta)$. The buyer accepts the offer if it does not exceed her reservation price. The reservation price satisfies the feasibility constraint $\rho_{K}^{U}(\eta, \widetilde{\eta}) \leq \eta$ and $\rho_{K}^{I}(\eta) \leq \eta$. $\widehat{V}_{K}(\eta)$ denotes the value function for an agent who is type- $K$ with money holdings of $\eta \cdot \mu(\eta)$ denotes the uninformed buyer's belief that a trading partner with money holdings of $\eta$ is the hightype. A stationary perfect Bayesian equilibrium with weakly undominated strategies consists of a time-invariant profile $<G_{H}, G_{L}, R^{I}, R^{U}, \Omega_{H y}^{H}, \Omega_{L y}^{H}, \Omega_{H y}^{L}, \Omega_{L y}^{L}, \Pi_{H y}, \Pi_{L y}, \omega_{H}, \omega_{L}, \rho_{H}^{I}, \rho_{L}^{I}, \rho_{H}^{U}, \rho_{L}^{U}, \mu>$ that satisfies: (1) Given that all agents play trading strategy $\left(\omega_{K}, \rho_{K}^{I}, \rho_{K}^{U}\right)$ and have a belief $\mu$, the distributions for money holdings $\left(G_{H}, G_{L}\right)$, reservation prices $\left(R^{I}, R^{U}\right)$ and offers $\left(\Omega_{K y}^{K^{\prime}}, \Pi_{K y}\right)$ are stationary. (2) Given the stationary distributions for money holdings $\left(G_{H}, G_{L}\right)$, reservation prices $\left(R^{I}, R^{U}\right)$ and offers $\left(\Omega_{K y}^{K^{\prime}}, \Pi_{K y}\right)$ and a belief $\mu$, the trading strategy 
$\left(\omega_{K}, \rho_{K}^{I}, \rho_{K}^{U}\right)$ is weakly undominated. The value function $\widehat{V}_{K}$ solves the following the Bellman equation.

$$
\begin{aligned}
& r \widehat{V_{K}}(\eta)= \\
& \alpha\left((1-\theta)\left\{\int\left[u+\widehat{V_{K}}\left(\eta-\omega_{H}(\widetilde{\eta})\right)-\widehat{V_{K}}(\eta)\right] d \Omega_{K \eta}^{H}(\widetilde{\eta})+\int\left[\widehat{V_{K}}\left(\eta-\omega_{L}(\widetilde{\eta})\right)-\widehat{V_{K}}(\eta)\right] d \Omega_{K \eta}^{L}(\widetilde{\eta})\right\}\right. \\
& +\theta \int\left[u+\widehat{V_{K}}\left(\eta-\omega_{H}(\widetilde{\eta})\right)-\widehat{V_{K}}(\eta)\right] d \Pi_{K \eta}(\widetilde{\eta}) \\
& \left.+\left\{(1-\theta)\left[1-R^{U}\left(\omega_{K}(\eta), \eta\right)\right]+1_{K} \theta\left[1-R^{I}\left(\omega_{H}(\eta)\right)\right]\right\}\left[\widehat{V_{K}}\left(\eta+\omega_{K}(\eta)\right)-\gamma-\widehat{V_{K}}(\eta)\right]\right)
\end{aligned}
$$

Since the reservation price of the informed buyer who meets an $L$-type seller is zero, for the low-type agents $\theta\left[1-R^{I}\left(\omega_{H}(\eta)\right)\right]$ is not in the last term.

Consider a single-price equilibrium, at which all trade occurs at a finite price $p$. Let $M_{K} p$ denote the maximum quantity of money that a type- $K$ agent holds in equilibrium. The support of money-holding distribution $G_{K}$ is $\left\{0, p, 2 p, \ldots, M_{K} p\right\}$. We conjecture an optimal profile for a single-price wealth-signaling equilibrium as follows.

(i) All informed buyers with money holdings of at least $p$ accept offer $p$ made by type- $H$ sellers, $\forall m=1,2, \ldots, \rho_{K}^{I}(m p) \geq p$.

(ii) Type- $K$ uninformed buyers with money holdings of $m p$ who meet sellers with money holdings of $\widetilde{m} p$ have strategies such that $\rho_{K}^{U}(m p, \widetilde{m} p) \geq p$ if $\left(m=1, \widetilde{m} \in\left[1, M_{H}-1\right]\right)$ or $\left(m \geq 2, \widetilde{m} \in\left[0, M_{H}-1\right]\right)$, and $\rho_{K}^{U}(m p, \tilde{m} p)<p$ if $(m, \widetilde{m})=(1,0)$ or $\left(m \geq 1, \widetilde{m} \geq M_{H}+1\right)$.

(iii) All sellers with money holdings of less than $M_{K} p$ offer price $p: \forall m=0,1,2, \ldots, M_{K}-1$, $\omega_{K}(m p)=p$.

(iv) Agents with money holdings greater than or equal to $M_{K} p$ offer above $p: \forall m \geq$ $M_{K}, \omega_{K}(m p)>p$.

(v) Offers made by type- $K$ agents with money holdings of $M_{K} p$ or more are not accepted 
by any buyer: $\forall m \geq M_{K}$,

$$
\omega_{K}(m p)>\max \left[\max _{l \leq M_{H}} \rho_{H}^{I}(l p), \max _{l \leq M_{H}} \rho_{H}^{U}(l p, m p), \max _{l \leq M_{L}} \rho_{L}^{I}(l p), \max _{l \leq M_{L}} \rho_{L}^{U}(l p, m p)\right]
$$

(vi) Type- $K$ sellers in state $M_{K}$ offer to sell at $Q_{K} p: \omega_{K}\left(M_{K} p\right)=Q_{K} p$.

(vii) There exists a least-money balance $R_{K} p\left(R_{K} \geq M_{K}\right)$ such that type- $K$ buyers who have money holdings greater than $R_{K} p$ and know a trading partner's type are willing to accept $Q_{H} p$. That is, $\rho_{K}^{I}(m p) \geq Q_{H} p$, and $\rho_{K}^{U}\left(m p, M_{H} p\right) \geq Q_{H} p$ if $M_{H}>M_{L},{ }^{9}$ if and only if $m>R_{K}$.

(viii) There exists a least-money balance $S_{K} p\left(S_{K} \geq R_{K}\right)$ such that type- $K$ buyers who have money holdings greater than $S_{K} p$ and do not know a trading partner's type are willing to accept $Q_{H} p$. That is, $\rho_{K}^{U}\left(m p, M_{H} p\right) \geq Q_{H} p$ when $M_{H}=M_{L}$ and $Q_{H}=Q_{L}$, if and only if $m>S_{K}$.

(ix) The uninformed buyer's belief that a trading partner with money holdings of $\eta$ is the high type is

$$
\mu(\eta)=\left\{\begin{array}{ccc}
\frac{d \Psi_{H}(\eta)}{d \Psi(\eta)} & \text { for } & d \Psi(\eta)>0 \\
0 & \text { for } & d \Psi(\eta)=0
\end{array}\right.
$$

where $\Psi_{H}(x)=G_{H}\{\eta \mid \eta \leq x\}$ and $\Psi(x)=G_{H}\{\eta \mid \eta \leq x\}+G_{L}\{\eta \mid \eta \leq x\}$.

We refer to a single-price wealth-signaling equilibrium at which all trades occur at price $p$, the support of money holdings distribution is $\{0, p, 2 p, \ldots, M p\}$ and all agents adopt the trading strategy profile (i)-(viii) and a belief (ix) as the first example of the wealth-signaling equilibrium (with $\left(M_{H}, M_{L}, Q_{H}, Q_{L}\right)$ ). Likewise, we conjecture an optimal profile for another single-price wealth-signaling equilibrium as: (i), (iii)-(ix) and (ii)' $\rho_{K}^{U}(m p, \widetilde{m} p) \geq p$ if ( $m \geq 1$, $\left.\widetilde{m} \in\left[1, M_{H}-1\right]\right)$ or $\left(m \geq T_{K}+1, \widetilde{m}=0\right)$, and $\rho_{K}^{U}(m p, \widetilde{m} p)<p$ if $\left(1 \leq m \leq T_{K}\right.$ and

\footnotetext{
${ }^{9}$ When $M_{H}>M_{L}$, the uninformed buyers know that the sellers with $M_{H} p$ money holdings are type- $H$ since they know the distribution of money. When $M_{H}<M_{L}$, the uninformed buyers know that the sellers who hold $M_{H} p$ money holdings and offer $Q_{H} p$ are type- $H$ since type- $L$ sellers with $M_{H} p$ money holdings offer price $p$. This fact also implies that $\rho_{K}^{U}(m p, \widetilde{m} p)=\rho_{K}^{I}(m p) \geq p$ if $M_{L}+1 \leq \widetilde{m} \leq M_{H}-1$, and $\rho_{K}^{U}(m p, \widetilde{m} p)=0$ if $M_{H}+1 \leq \widetilde{m} \leq M_{L}-1$.
} 
$\widetilde{m}=0)$ or $\left(m \geq 1, \widetilde{m} \geq M_{H}+1\right)$, for some $T_{K}\left(\geq M_{K}\right)$. We refer to this equilibrium as the second example of the wealth-signaling equilibrium. We also conjecture an optimal profile for a single-price non-wealth-signaling equilibrium as: (i), (iii)-(ix) and (ii)" $\rho_{K}^{U}(m p, \widetilde{m} p) \geq p$ for $\left(m \geq 1, \widetilde{m} \in\left[0, M_{H}-1\right]\right)$, and $\rho_{K}^{U}(m p, \widetilde{m} p)<p$ for $\left(m \geq 1, \widetilde{m} \geq M_{H}+1\right)$.

Define $V_{K}(\lfloor\eta / p\rfloor)=\widehat{V}_{K}(\eta)$ (where $\lfloor x\rfloor$ is the integer part of $x$ ) and $P_{m}^{K}=d G_{K}(m p)$. Define $\sigma_{K}(m, \tilde{m})$ as the probability that a type- $K$ uninformed buyer who holds money holdings of $m p$ (hereafter, $m$ units of money) accepts the offer by a seller with money holdings of $\widetilde{m} p$. If $\rho_{K}^{U}(m p, \widetilde{m} p) \geq \omega_{K^{\prime}}(\widetilde{m} p)$, then $\sigma_{K}(m, \tilde{m})=1$, and if $\rho_{K}^{U}(m p, \widetilde{m} p)<\omega_{K^{\prime}}(\widetilde{m} p)$, then $\sigma_{K}(m, \tilde{m})=0$, when an agent unidentifies the trading partner's type. On the wealth-signaling and non-wealth-signaling equilibria with $\left(M_{H}, M_{L}, Q_{H}, Q_{L}\right)$, the Bellman equation (8) can be written as:

For $m=0$, equation $(2) \cdot{ }^{10}$

For $m=1,2, \ldots, M_{K}-1$, equation (1).

For $m=M_{K}, \ldots, R_{K}$,

$$
\begin{aligned}
& r V_{K}(m) \\
& =\alpha\left\{(1-\theta) \sum_{\widetilde{m}=0}^{M-1} P_{\widetilde{m}} \sigma_{K}(m, \widetilde{m})\left[\mu_{\widetilde{m}} u+V_{K}(m-1)-V_{K}(m)\right]\right. \\
& \left.+\theta \sum_{\widetilde{m}=0}^{M-1} P_{\widetilde{m}}^{H}\left[u+V_{K}(m-1)-V_{K}(m)\right]\right\}
\end{aligned}
$$

\footnotetext{
${ }^{10} \phi_{K}(m)=\Phi_{K}(m)=1$ and $\sigma_{K}(m, \widetilde{m})=\Sigma_{K}(m, \widetilde{m})$.
} 
For $m=R_{K}+1, \ldots, S_{K}$,

$$
\begin{aligned}
& r V_{K}(m) \\
& =\alpha\left\{(1-\theta) \sum_{\tilde{m}=0}^{M-1} P_{\widetilde{m}} \sigma_{K}(m, \widetilde{m})\left[\mu_{\widetilde{m}} u+V_{K}(m-1)-V_{K}(m)\right]\right. \\
& +\theta \sum_{\widetilde{m}=0}^{M-1} P_{\widetilde{m}}^{H}\left[u+V_{K}(m-1)-V_{K}(m)\right] \\
& \left.+[1-\chi(1-\theta)] P_{M_{H}}^{H}\left[u+V_{K}\left(m-Q_{H}\right)-V_{K}(m)\right]\right\}
\end{aligned}
$$

For $m \geq S_{K}+1$,

$$
\begin{aligned}
& r V_{K}(m) \\
& =\alpha\left\{(1-\theta) \sum_{\tilde{m}=0}^{M-1} P_{\tilde{m}} \sigma_{K}(m, \widetilde{m})\left[\mu_{\tilde{m}} u+V_{K}(m-1)-V_{K}(m)\right]\right. \\
& +\theta \sum_{\tilde{m}=0}^{M-1} P_{\tilde{m}}^{H}\left[u+V_{K}(m-1)-V_{K}(m)\right] \\
& +[1-\chi(1-\theta)] P_{M_{H}}^{H}\left[u+V_{K}\left(m-Q_{H}\right)-V_{K}(m)\right] \\
& \left.+\chi(1-\theta) P_{M_{H}}\left[\mu_{M_{H}} u+V_{K}\left(m-Q_{H}\right)-V_{K}(m)\right]\right\},
\end{aligned}
$$

where $\chi=1$ if $M_{H}=M_{L}$ and 0 otherwise.

The population equations are the same as equations (4)-(7). Equations (9)-(11) describe the value functions off the equilibrium path. The value functions presented in Section 2 thus correspond with those on the equilibrium path in this section. The conditions under which the conjectured equilibrium trading profile (i)-(viii) and a belief (ix) are optimal are summarized in the following lemma.

LEMMA1. For a given set of the parameters, $\bar{M}, u, \gamma, \alpha, r$ and $\theta$ : 
(a) Suppose that $V_{K}(3)-V_{K}(2) \leq \gamma<V_{K}(2)-V_{K}(1) \leq \mu_{0} u<V_{K}(1)-V_{K}(0) \leq \mu_{1} u$

$$
\begin{gathered}
\text { If } V_{K}(4)-V_{K}(2)>\gamma \text {, then } V_{K}(2)-V_{K}(0)>u \\
\text { If } R_{K}=3 \text {, then } V_{K}(4)-V_{K}(3) \leq \gamma \\
\max \left\{\theta P_{2}^{H}\left(P_{\tilde{m}}^{L}-P_{\tilde{m}}^{H}\right) / P_{\tilde{m}}^{H},(1-\theta)\left[P_{2}^{H}\left(P_{\tilde{m}}^{L} / P_{\tilde{m}}^{H}\right)-P_{2}^{L}\right], \theta P_{2}^{L}\right\} \leq r / \alpha
\end{gathered}
$$

for $K=H, L$ and $\widetilde{m}=0,1$.

Then there exits the first example of the wealth-signaling equilibrium where $M_{K}=Q_{K}=2$ (for $K=H, L)$.

(b) Suppose that $\max \left(\gamma, \mu_{0} u\right)<V_{K}(1)-V_{K}(0) \leq \mu_{1} u$, $\max \left(\gamma, \mu_{0} u\right)<V_{K}(2)-V_{K}(1) \leq \mu_{1} u$, $V_{K}(3)-V_{K}(2) \leq \gamma$, and $(1-\theta) P_{0}^{H} \leq r / \alpha$. Suppose $P_{2}^{H}\left(P_{0}^{L}-P_{0}^{H}\right) / P_{0}^{H} \leq r / \alpha$ when $T_{K} \leq R_{K}$, $P_{2}^{H} P_{0}^{L} / P_{0}^{H} \leq r / \alpha$ when $T_{K}>R_{K}$, and the conditions (12) and (13) hold. Then there exits the second example of the wealth-signaling equilibrium where $M_{K}=Q_{K}=2$.

(c) If $\gamma<V_{K}(1)-V_{K}(0) \leq \min \left(\mu_{0} u, \mu_{1} u\right), V_{K}(3)-V_{K}(2) \leq \gamma<V_{K}(2)-V_{K}(1) \leq$ $\min \left(\mu_{0} u, \mu_{1} u\right)$, and the conditions (12), (13) and (14) hold, then there exists a non-wealthsignaling equilibrium where $M_{K}=Q_{K}=2$.

The proof is shown in Appendix. It refers to Zhou's (1999) proof.

The existence regions of these wealth-signaling and non-wealth-signaling equilibria are smaller than their counterparts for the equilibria studied in Section 3 due to the additional conditions. The same interpretation as in Section 3 is, however, applicable to these regions. Figure 5 shows the existence region of the first example of the wealth-signaling equilibrium. ${ }^{11}$ The distributions of money are identical with the counterparts in Section 3. The numerical comparison of the welfare levels also attains the same interpretation as in Section 3.

$<<$ Insert Figure $5>>$

To focus on the main conclusion that the seller's money holdings can signal the quality of

\footnotetext{
${ }^{11}$ The regions of other equilibria are available from the author upon request.
} 
her products, the only seller's money holdings are assumed observable. However, the buyer's are assumed not observable in this section. If the buyers' money holdings are observable, the sellers' offer may depend not only on their own money holdings but also on those of the trading partners. In this case, poor sellers may offer a lower price only to poor uninformed buyers (with a high marginal valuation of money) so that they can buy. ${ }^{12}$

\section{Conclusion}

This paper has studied the possibility that wealth (holdings of money) serves as a signal of ability as a producer when not all agents can directly observe a producer's ability to produce high-quality products. Ability is directly observed by some agents; hence an agent's wealth may advertise her past performance in selling products. The framework is a combination of signaling models and Williamson and Wright's (1994) search-theoretic model of money, but this approach assumes that an agent has imperfect knowledge of the trading partner's innate ability and can hold multiple units of money. The paper shows that such signaling equilibria exist. Agents save more (or spend less) money to signal their ability as producers, and those with wealth increase sales more easily than those without. As a consequence, wealth is more unequally distributed than in an economy where agents buy regardless of producers' wealth. Wealth signaling has the positive and negative effects on welfare. The informational effect helps agents better identify product quality; the distributional effect, however, decreases the opportunity for trade. A numerical comparison of welfare levels shows that this unfavorable effect on welfare is dominant for a large part of the co-existence region.

\footnotetext{
${ }^{12}$ Camera and Corbae (1999) studied equilibrium price dispersion in the economy where both sellers and buyers observe the trading partners' money holdings and showed the numerical examples of heterogeneous exchange patterns across agents of different money holdings.
} 


\section{APPENDIX}

\section{PROOF OF LEMMA 1.}

First, we present the set of weakly undominated offers and the sets of weakly undominated reservation prices of informed and uninformed agents.

LEMMA 2. For an agent with money holdings $\eta \in \Re_{+}$, the set of weakly undominated offers

$A_{K}(\eta)$ and the sets of weakly undominated reservation prices of informed and uninformed agents, $B_{K}^{I}(\eta)$ and $B_{K}^{U}(\eta, \widetilde{\eta})$, respectively for $K=H, L$ are:

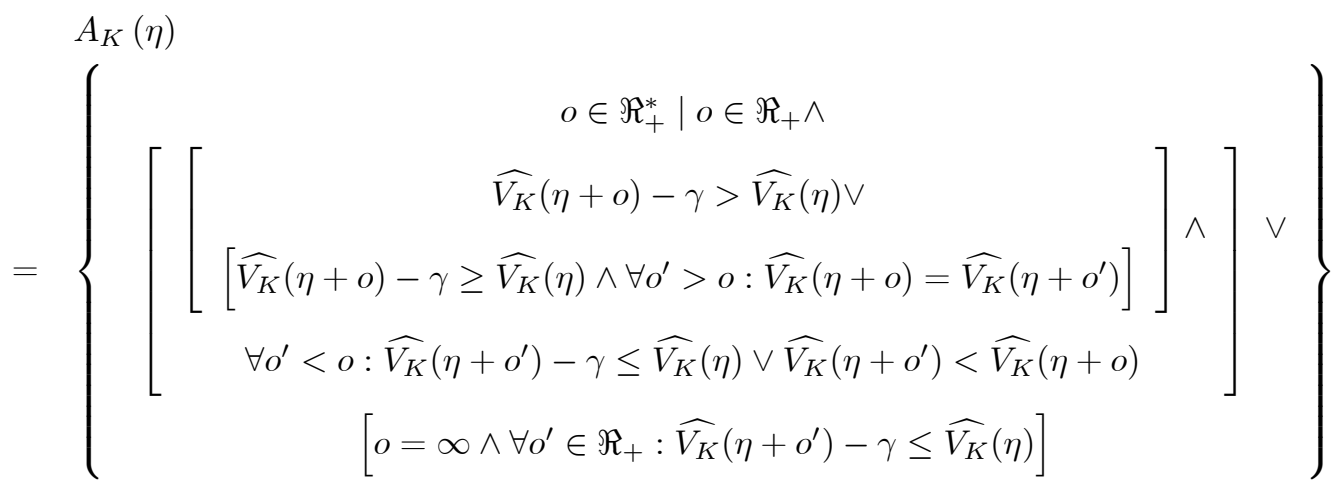

$$
=\left\{r \in[0, \eta] \mid\left[\begin{array}{c}
\forall r^{\prime}>r: u+\widehat{V_{K}}\left(\eta-r^{\prime}\right) \leq \widehat{V_{K}}(\eta) \wedge \\
u+\widehat{V_{K}}(\eta-r) \geq \widehat{V_{K}}(\eta) \vee \\
\forall r^{\prime}<r\left[\exists o \in\left(r^{\prime}, r\right]: u+\widehat{V_{K}}(\eta-o)>\widehat{V_{K}}(\eta)\right]
\end{array}\right]\right\}
$$




$$
=\left\{r \in[0, \eta] \mid\left[\begin{array}{c}
\forall r^{\prime}>r: u \mu(\widetilde{\eta})+\widehat{V_{K}}\left(\eta-r^{\prime}\right)-\widehat{V_{K}}(\eta) \leq 0 \wedge \\
u \mu(\widetilde{\eta})+\widehat{V_{K}}(\eta-r)-\widehat{V_{K}}(\eta) \geq 0 \vee \\
\forall r^{\prime}<r:\left[\exists o \in\left(r^{\prime}, r\right]: u \mu(\widetilde{\eta})+\widehat{V_{K}}(\eta-o)-\widehat{V_{K}}(\eta)>0\right]
\end{array}\right]\right\}
$$

, where $\mu(\widetilde{\eta})$ is the uninformed buyer's belief that a trading partner with money holdings of $\widetilde{\eta}$ is a high type.

Proof. Define $\Lambda_{s K}\left(\eta_{s}, o, r\right)=\widehat{V_{K}}\left(\eta_{s}+o\right)-\gamma-\widehat{V_{K}}\left(\eta_{s}\right)$ if $o \leq r$ and 0 otherwise. $\Lambda_{b K}^{I}\left(\eta_{b}, o, r\right)=u+\widehat{V_{K}}\left(\eta_{b}-o\right)-\widehat{V_{K}}\left(\eta_{b}\right)$ if $o \leq r$ and 0 otherwise. $\Lambda_{b K}^{U}\left(\eta_{b}, \widetilde{\eta_{s}}, o, r\right)=$ $u \mu\left(\widetilde{\eta_{s}}\right)+\widehat{V_{K}}\left(\eta_{b}-o\right)-\widehat{V_{K}}\left(\eta_{b}\right)$ if $o \leq r$ and 0 otherwise.

Consider the offer first: $o \in A_{K}(\eta) \Leftrightarrow o$ is weakly undominated.

" $\Rightarrow$ ": Take an offer $o \in A_{K}(\eta)$.

Suppose that $o$ is weakly dominated by another strategy $o^{\prime}$.

If $o \in \Re_{+}$and $o^{\prime}<o$, then either (a) $\widehat{V_{K}}\left(\eta+o^{\prime}\right)-\gamma \leq \widehat{V_{K}}(\eta) \vee(\mathrm{b}) \widehat{V_{K}}\left(\eta+o^{\prime}\right)<\widehat{V_{K}}(\eta+o)$.

Consider (a). For all $r<o^{\prime}, \Lambda_{s K}\left(\eta, o^{\prime}, r\right)=\Lambda_{s K}(\eta, o, r)=0$.

For all $r \in\left(o^{\prime}, o\right), \Lambda_{s K}\left(\eta, o^{\prime}, r\right)=\widehat{V_{K}}\left(\eta+o^{\prime}\right)-\gamma-\widehat{V_{K}}(\eta) \leq 0=\Lambda_{s K}(\eta, o, r)$. For all $r \geq o$, $\Lambda_{s K}\left(\eta, o^{\prime}, r\right)=\widehat{V_{K}}\left(\eta+o^{\prime}\right)-\gamma-\widehat{V_{K}}(\eta) \leq \widehat{V_{K}}(\eta+o)-\gamma-\widehat{V_{K}}(\eta)=\Lambda_{s K}(\eta, o, r)$. Thus, there does not exist an $r$ such that $\Lambda_{s K}\left(\eta, o^{\prime}, r\right)>\Lambda_{s K}(\eta, o, r)$. Hence, the offer $o$ is not weakly dominated by $o^{\prime}$.

Consider (b). For all $r \geq o, \Lambda_{s K}\left(\eta, o^{\prime}, r\right)=\widehat{V_{K}}\left(\eta+o^{\prime}\right)-\gamma-\widehat{V_{K}}(\eta)<\widehat{V_{K}}(\eta+o)-\gamma-\widehat{V_{K}}(\eta)=$ $\Lambda_{s K}(\eta, o, r)$.

Then, $o$ is not weakly dominated by $o^{\prime}$.

If $o \in \Re_{+}$and $o^{\prime}>o$, then (i) $\widehat{V_{K}}(\eta+o)-\gamma>\widehat{V_{K}}(\eta) \vee$ (ii) $\widehat{V_{K}}(\eta+o)-\gamma \geq \widehat{V_{K}}(\eta) \wedge$ $\widehat{V_{K}}\left(\eta+o^{\prime}\right)=\widehat{V_{K}}(\eta+o)$. When $(\mathrm{i}) \widehat{V_{K}}(\eta+o)-\gamma>\widehat{V_{K}}(\eta)$, for all $r \in\left(o, o^{\prime}\right), \Lambda_{s K}(\eta, o, r)=$ $\widehat{V_{K}}(\eta+o)-\gamma-\widehat{V_{K}}(\eta)>0=\Lambda_{s K}\left(\eta, o^{\prime}, r\right)$. Hence, $o$ is not weakly dominated by $o^{\prime}$. When (ii) $\widehat{V_{K}}(\eta+o)-\gamma \geq \widehat{V_{K}}(\eta) \wedge \widehat{V_{K}}\left(\eta+o^{\prime}\right)=\widehat{V_{K}}(\eta+o)$, for all $r<o, \Lambda_{s K}\left(\eta, o^{\prime}, r\right)=\Lambda_{s K}(\eta, o, r)=0$, and for all $r \in\left(o, o^{\prime}\right), \Lambda_{s K}(\eta, o, r)=\widehat{V_{K}}(\eta+o)-\gamma-\widehat{V_{K}}(\eta) \geq 0=\Lambda_{s K}\left(\eta, o^{\prime}, r\right)$. For all $o^{\prime} \leq r$, 
$\Lambda_{s K}(\eta, o, r)=\widehat{V_{K}}(\eta+o)-\gamma-\widehat{V_{K}}(\eta)=\widehat{V_{K}}\left(\eta+o^{\prime}\right)-\gamma-\widehat{V_{K}}(\eta)=\Lambda_{s K}\left(\eta, o^{\prime}, r\right)$. Hence, there does not exist an $r$ such that $\Lambda_{s K}\left(\eta, o^{\prime}, r\right)>\Lambda_{s K}(\eta, o, r)$. Hence, $o$ is not weakly dominated by $o^{\prime}$.

If $o=\infty \wedge \widehat{V_{K}}\left(\eta+o^{\prime}\right)-\gamma \leq \widehat{V_{K}}(\eta)$, then for all $r<o^{\prime}, \Lambda_{s K}\left(\eta, o^{\prime}, r\right)=\Lambda_{s K}(\eta, o, r)=0$ and for all $r \in\left(o^{\prime}, \infty\right), \Lambda_{s K}\left(\eta, o^{\prime}, r\right)=\widehat{V_{K}}\left(\eta+o^{\prime}\right)-\gamma-\widehat{V_{K}}(\eta) \leq 0=\Lambda_{s K}(\eta, o, r)$. Hence, there does not exist an $r$ such that $\Lambda_{s K}\left(\eta, o^{\prime}, r\right)>\Lambda_{s K}(\eta, o, r)$. Hence, $o$ is not weakly dominated by $o^{\prime}$.

" $\Leftarrow$ : Take a weakly undominated offer $o$ and suppose that $o \notin A_{K}(\eta)$.

(a) $o \in \Re_{+} \wedge \widehat{V_{K}}(\eta+o)-\gamma<\widehat{V_{K}}(\eta)$. Take $o^{\prime}>o$. For all $r<o, \Lambda_{s K}\left(\eta, o^{\prime}, r\right)=\Lambda_{s K}(\eta, o, r)=$ 0 . For all $r \in\left(o, o^{\prime}\right), \Lambda_{s K}(\eta, o, r)=\widehat{V_{K}}(\eta+o)-\gamma-\widehat{V_{K}}(\eta)<0=\Lambda_{s K}\left(\eta, o^{\prime}, r\right)$. For all $r \geq o^{\prime}$, $\Lambda_{s K}(\eta, o, r)=\widehat{V_{K}}(\eta+o)-\gamma-\widehat{V_{K}}(\eta) \leq \widehat{V_{K}}\left(\eta+o^{\prime}\right)-\gamma-\widehat{V_{K}}(\eta)=\Lambda_{s K}\left(\eta, o^{\prime}, r\right)$. Hence, $o^{\prime}$ weakly dominates $o$. (b) $o \in \Re_{+} \wedge \widehat{V_{K}}(\eta+o)-\gamma \leq \widehat{V_{K}}(\eta) \wedge \exists o^{\prime}>o: \widehat{V_{K}}(\eta+o)<\widehat{V_{K}}\left(\eta+o^{\prime}\right)$. For all $r<o$, $\Lambda_{s K}\left(\eta, o^{\prime}, r\right)=\Lambda_{s K}(\eta, o, r)=0$. For all $r \in\left(o, o^{\prime}\right), \Lambda_{s K}(\eta, o, r)=\widehat{V_{K}}(\eta+o)-\gamma-\widehat{V_{K}}(\eta) \leq 0=$ $\Lambda_{s K}\left(\eta, o^{\prime}, r\right)$. For all $r \geq o^{\prime}, \Lambda_{s K}(\eta, o, r)=\widehat{V_{K}}(\eta+o)-\gamma-\widehat{V_{K}}(\eta)<\widehat{V_{K}}\left(\eta+o^{\prime}\right)-\gamma-\widehat{V_{K}}(\eta)=$ $\Lambda_{s K}\left(\eta, o^{\prime}, r\right)$. Hence, $o^{\prime}$ weakly dominates $o$. (c) $o \in \Re_{+} \wedge \exists o^{\prime}<o: \widehat{V_{K}}\left(\eta+o^{\prime}\right)-\gamma>\widehat{V_{K}}(\eta) \wedge$ $\widehat{V_{K}}\left(\eta+o^{\prime}\right)=\widehat{V_{K}}(\eta+o)$. For all $r<o^{\prime}, \Lambda_{s K}\left(\eta, o^{\prime}, r\right)=\Lambda_{s K}(\eta, o, r)=0$. For all $r \in\left(o^{\prime}, o\right)$, $\Lambda_{s K}\left(\eta, o^{\prime}, r\right)=\widehat{V_{K}}\left(\eta+o^{\prime}\right)-\gamma-\widehat{V_{K}}(\eta)>0=\Lambda_{s K}(\eta, o, r)$. For all $o \leq r, \Lambda_{s K}\left(\eta, o^{\prime}, r\right)=$ $\widehat{V_{K}}\left(\eta+o^{\prime}\right)-\gamma-\widehat{V_{K}}(\eta)=\widehat{V_{K}}(\eta+o)-\gamma-\widehat{V_{K}}(\eta)=\Lambda_{s K}(\eta, o, r)$. Hence, $o^{\prime}$ weakly dominates $o$. (d) $o=\infty \wedge \exists o^{\prime} \in \Re_{+}: \widehat{V_{K}}\left(\eta+o^{\prime}\right)-\gamma>\widehat{V_{K}}(\eta)$. For all $r<o^{\prime}, \Lambda_{s K}\left(\eta, o^{\prime}, r\right)=\Lambda_{s K}(\eta, o, r)=0$. For all $r \geq o^{\prime}, \Lambda_{s K}\left(\eta, o^{\prime}, r\right)=\widehat{V_{K}}\left(\eta+o^{\prime}\right)-\gamma-\widehat{V_{K}}(\eta)>0=\Lambda_{s K}(\eta, o, r)$. Hence, $o^{\prime}$ weakly dominates $o$.

The results of all four cases contradict the assumption that offer $o$ is weakly undominated. Hence $o \in A_{K}(\eta)$. This concludes the proof that $o$ is weakly undominated if and only if $o \in$ $A_{K}(\eta)$.

Next, consider the reservation price of the informed agents: $r \in B_{K}^{I}(\eta) \Leftrightarrow r$ is weakly undominated.

" $\Rightarrow$ " : Take $r \in B_{K}^{I}(\eta)$ and suppose that $r$ is weakly dominated by $r^{\prime}$. Consider (a) $r^{\prime}<r$. For all $o \leq r^{\prime}, \Lambda_{b K}^{I}(\eta, o, r)=u+\widehat{V_{K}}(\eta-o)-\widehat{V_{K}}(\eta)=\Lambda_{b K}^{I}\left(\eta, o, r^{\prime}\right)$. For all $o>r$, 
$\Lambda_{b K}^{I}(\eta, o, r)=\Lambda_{b K}^{I}\left(\eta, o, r^{\prime}\right)=0$. For $o \in\left(r^{\prime}, r\right], r \in B_{K}^{I}(\eta) \Longrightarrow(i) u+\widehat{V_{K}}(\eta-r) \geq \widehat{V_{K}}(\eta) \vee$ (ii) $\forall r^{\prime}<r: \exists o \in\left(r^{\prime}, r\right]: u+\widehat{V_{K}}(\eta-o)>\widehat{V_{K}}(\eta)$. When (i) $u+\widehat{V_{K}}(\eta-r) \geq \widehat{V_{K}}(\eta)$, for all $o \in\left(r^{\prime}, r\right], \Lambda_{b K}^{I}(\eta, o, r)=u+\widehat{V_{K}}(\eta-o)-\widehat{V_{K}}(\eta) \geq u+\widehat{V_{K}}(\eta-r)-\widehat{V_{K}}(\eta) \geq 0=\Lambda_{b K}^{I}\left(\eta, o, r^{\prime}\right)$. Hence there does not exist $o \in \Re_{+}^{*}$ such that $\Lambda_{b K}^{I}(\eta, o, r)<\Lambda_{b K}^{I}\left(\eta, o, r^{\prime}\right)$. Hence $r$ is not weakly dominated by $r^{\prime}$. When (ii) $\forall r^{\prime}<r: \exists o \in\left(r^{\prime}, r\right]: u+\widehat{V_{K}}(\eta-o)>\widehat{V_{K}}(\eta), \Lambda_{b K}^{I}(\eta, o, r)=$ $u+\widehat{V_{K}}(\eta-o)-\widehat{V_{K}}(\eta)>0=\Lambda_{b K}^{I}\left(\eta, o, r^{\prime}\right)$. Hence $r$ is not weakly dominated by $r^{\prime}$.

Consider (b) $r<r^{\prime}$. For all $o \leq r, \Lambda_{b K}^{I}(\eta, o, r)=u+\widehat{V_{K}}(\eta-o)-\widehat{V_{K}}(\eta)=\Lambda_{b K}^{I}\left(\eta, o, r^{\prime}\right)$. For all $o>r^{\prime}, \Lambda_{b K}^{I}(\eta, o, r)=\Lambda_{b K}^{I}\left(\eta, o, r^{\prime}\right)=0$. For $o \in\left(r, r^{\prime}\right], \Lambda_{b K}^{I}(\eta, o, r)=0 \geq u+\widehat{V_{K}}(\eta-$ $o)-\widehat{V_{K}}(\eta)=\Lambda_{b K}^{I}\left(\eta, o, r^{\prime}\right)$. There does not exist $o \in \Re_{+}^{*}$ such that $\Lambda_{b K}^{I}(\eta, o, r)<\Lambda_{b K}^{I}\left(\eta, o, r^{\prime}\right)$. Hence $r$ is not weakly dominated by $r^{\prime}$.

" $\Leftarrow "$ : Take a weakly undominated $r$ and suppose that $r \notin B_{K}^{I}(\eta)$. Then, $r \notin B_{K}^{I}(\eta) \Rightarrow$ (i) $\exists r^{\prime}>r: u+\widehat{V_{K}}\left(\eta-r^{\prime}\right)>\widehat{V_{K}}(\eta) \vee$ (ii) $u+\widehat{V_{K}}(\eta-r)<\widehat{V_{K}}(\eta) \wedge \exists r^{\prime}<r: \forall o \in\left(r^{\prime}, r\right]$ : $u+\widehat{V_{K}}(\eta-o) \leq \widehat{V_{K}}(\eta)$.

Consider (i). For $o<r, \Lambda_{b K}^{I}(\eta, o, r)=\Lambda_{b K}^{I}(\eta, o, \widehat{r})=u+\widehat{V_{K}}(\eta-o)-\widehat{V_{K}}(\eta)$. For all $o \in(\widehat{r}, \infty), \Lambda_{b K}^{I}(\eta, o, r)=\Lambda_{b K}^{I}(\eta, o, \widehat{r})=0$. For all $o \in(r, \widehat{r}), \widehat{V_{K}}(\eta-o) \geq \widehat{V_{K}}(\eta-\widehat{r})$. Hence, $\Lambda_{b K}^{I}(\eta, o, \widehat{r})=u+\widehat{V_{K}}(\eta-o)-\widehat{V_{K}}(\eta) \geq u+\widehat{V_{K}}(\eta-\widehat{r})-\widehat{V_{K}}(\eta)>0=\Lambda_{b K}^{I}(\eta, o, r)$. Hence $\widehat{r}$ weakly dominates $r$. Consider (ii). For all $o \leq r^{\prime}, \Lambda_{b K}^{I}(\eta, o, r)=u+\widehat{V_{K}}(\eta-o)-\widehat{V_{K}}(\eta)=\Lambda_{b K}^{I}\left(\eta, o, r^{\prime}\right)$. For all $r<o, \Lambda_{b K}^{I}(\eta, o, r)=\Lambda_{b K}^{I}\left(\eta, o, r^{\prime}\right)=0$. For all $o \in\left(r^{\prime}, r\right), \Lambda_{b K}^{I}(\eta, o, r)=u+\widehat{V_{K}}(\eta-o)-$ $\widehat{V_{K}}(\eta) \leq 0=\Lambda_{b K}^{I}\left(\eta, o, r^{\prime}\right)$. For $o=r, \Lambda_{b K}^{I}(\eta, r, r)=u+\widehat{V_{K}}(\eta-r)-\widehat{V_{K}}(\eta)<0=\Lambda_{b K}^{I}\left(\eta, r, r^{\prime}\right)$. Hence, $r^{\prime}$ weakly dominates $r$. Since (i) and (ii) contradict the assumption that $r$ is weakly undominated, $r \in B_{K}^{I}(\eta)$.

Finally, consider the reservation price of the uninformed agents: $r \in B_{K}^{U}(\eta, \widetilde{\eta}) \Leftrightarrow r$ is weakly undominated.

" $\Rightarrow$ " : Take $r \in B_{K}^{U}(\eta, \widetilde{\eta})$ and suppose that $r$ is weakly dominated by $r^{\prime}$. Consider (a) $r^{\prime}<r$. For all $o \leq r^{\prime}, \Lambda_{b K}^{U}(\eta, \widetilde{\eta}, o, r)=u \mu(\widetilde{\eta})+\widehat{V_{K}}(\eta-o)-\widehat{V_{K}}(\eta)=\Lambda_{b K}^{U}\left(\eta, \widetilde{\eta}, o, r^{\prime}\right)$. For all $o>r, \Lambda_{b K}^{U}(\eta, \widetilde{\eta}, o, r)=\Lambda_{b K}^{U}\left(\eta, \widetilde{\eta}, o, r^{\prime}\right)=0$. For $o \in\left(r^{\prime}, r\right], r \in B_{K}^{U}(\eta, \widetilde{\eta}) \Longrightarrow(i) u \mu(\widetilde{\eta})+$ $\widehat{V_{K}}(\eta-r)-\widehat{V_{K}}(\eta) \geq 0 \vee(i i) \forall r^{\prime}<r: \exists o \in\left(r^{\prime}, r\right]: u \mu(\widetilde{\eta})+\widehat{V_{K}}(\eta-o)-\widehat{V_{K}}(\eta)>0$. When (i) 
$u \mu(\widetilde{\eta})+\widehat{V_{K}}(\eta-r)-\widehat{V_{K}}(\eta) \geq 0$, for all $o \in\left(r^{\prime}, r\right], \Lambda_{b K}^{U}(\eta, \widetilde{\eta}, o, r)=u \mu(\widetilde{\eta})+\widehat{V_{K}}(\eta-o)-\widehat{V_{K}}(\eta) \geq$ $u \mu(\widetilde{\eta})+\widehat{V_{K}}(\eta-r)-\widehat{V_{K}}(\eta) \geq 0=\Lambda_{b K}^{U}\left(\eta, \widetilde{\eta}, o, r^{\prime}\right)$. Hence there does not exist $o \in \Re_{+}^{*}$ such that $\Lambda_{b K}^{U}(\eta, \widetilde{\eta}, o, r)<\Lambda_{b K}^{U}\left(\eta, \widetilde{\eta}, o, r^{\prime}\right)$. Hence $r$ is not weakly dominated by $r^{\prime}$. When (ii) $\forall r^{\prime}<r$ : $\exists o \in\left(r^{\prime}, r\right]: u \mu(\widetilde{\eta})+\widehat{V_{K}}(\eta-o)-\widehat{V_{K}}(\eta)>0, \Lambda_{b K}^{U}(\eta, \widetilde{\eta}, o, r)=u \mu(\widetilde{\eta})+\widehat{V_{K}}(\eta-o)-\widehat{V_{K}}(\eta)>$ $0=\Lambda_{b K}^{U}\left(\eta, \widetilde{\eta}, o, r^{\prime}\right)$. Hence $r$ is not weakly dominated by $r^{\prime}$.

Consider (b) $r<r^{\prime}$. For all $o \leq r, \Lambda_{b K}^{U}(\eta, \widetilde{\eta}, o, r)=u \mu(\widetilde{\eta})+\widehat{V_{K}}(\eta-o)-\widehat{V_{K}}(\eta)=$ $\Lambda_{b K}^{U}\left(\eta, \widetilde{\eta}, o, r^{\prime}\right)$. For all $o>r^{\prime}, \Lambda_{b K}^{U}(\eta, \widetilde{\eta}, o, r)=\Lambda_{b K}^{U}\left(\eta, \widetilde{\eta}, o, r^{\prime}\right)=0$. For all $o \in\left(r, r^{\prime}\right]$, $\Lambda_{b K}^{U}(\eta, \widetilde{\eta}, o, r)=0 \geq u \mu(\widetilde{\eta})+\widehat{V_{K}}(\eta-o)-\widehat{V_{K}}(\eta)=\Lambda_{b K}^{U}\left(\eta, \widetilde{\eta}, o, r^{\prime}\right)$. There does not exist $o \in \Re_{+}^{*}$ such that $\Lambda_{b K}^{U}(\eta, \widetilde{\eta}, o, r)<\Lambda_{b K}^{U}\left(\eta, \widetilde{\eta}, o, r^{\prime}\right)$. Hence $r$ is not weakly dominated by $r^{\prime}$.

" $\Leftarrow$ " : Take a weakly undominated $r$ and suppose that $r \notin B_{K}^{U}(\eta, \widetilde{\eta})$. Then, $r \notin B_{K}^{U}(\eta, \widetilde{\eta}) \Rightarrow$ (i) $\exists \widehat{r}>r: u \mu(\widetilde{\eta})+\widehat{V_{K}}(\eta-\widehat{r})-\widehat{V_{K}}(\eta)>0 \vee$ (ii) $u \mu(\widetilde{\eta})+\widehat{V_{K}}(\eta-r)-\widehat{V_{K}}(\eta)<0 \wedge \exists r^{\prime}<r$ : $\forall o \in\left(r, r^{\prime}\right]: u \mu(\widetilde{\eta})+\widehat{V_{K}}(\eta-o)-\widehat{V_{K}}(\eta) \leq 0$.

Consider (i). For $o<r, \Lambda_{b K}^{U}(\eta, \widetilde{\eta}, o, r)=\Lambda_{b K}^{U}(\eta, \widetilde{\eta}, o, \widehat{r})=u \mu(\widetilde{\eta})+\widehat{V_{K}}(\eta-o)-\widehat{V_{K}}(\eta)$. For all $o \in(\widehat{r}, \infty), \Lambda_{b K}^{U}(\eta, \widetilde{\eta}, o, r)=\Lambda_{b K}^{U}(\eta, \widetilde{\eta}, o, \widehat{r})=0$. For all $o \in(r, \widehat{r}), \Lambda_{b K}^{U}(\eta, \widetilde{\eta}, o, \widehat{r})=$ $u \mu(\widetilde{\eta})+\widehat{V_{K}}(\eta-o)-\widehat{V_{K}}(\eta) \geq u \mu(\widetilde{\eta})+\widehat{V_{K}}(\eta-\widehat{r})-\widehat{V_{K}}(\eta)>0=\Lambda_{b K}^{U}(\eta, \widetilde{\eta}, o, r)$. Hence $\widehat{r}$ weakly dominates $r$. Consider (ii). For all $o \leq r^{\prime}, \Lambda_{b K}^{U}(\eta, \widetilde{\eta}, o, r)=u \mu(\widetilde{\eta})+\widehat{V_{K}}(\eta-o)-$ $\widehat{V_{K}}(\eta)=\Lambda_{b K}^{U}\left(\eta, \widetilde{\eta}, o, r^{\prime}\right)$. For all $r<o, \Lambda_{b K}^{U}(\eta, \widetilde{\eta}, o, r)=\Lambda_{b K}^{U}\left(\eta, \widetilde{\eta}, o, r^{\prime}\right)=0$. For all $o \in\left(r^{\prime}, r\right)$, $\Lambda_{b K}^{U}(\eta, \widetilde{\eta}, o, r)=u \mu(\widetilde{\eta})+\widehat{V_{K}}(\eta-o)-\widehat{V_{K}}(\eta) \leq 0=\Lambda_{b K}^{U}\left(\eta, \widetilde{\eta}, o, r^{\prime}\right)$. For $o=r, \Lambda_{b K}^{U}(\eta, \widetilde{\eta}, r, r)=$ $u \mu(\widetilde{\eta})+\widehat{V_{K}}(\eta-r)-\widehat{V_{K}}(\eta)<0=\Lambda_{b K}^{U}\left(\eta, \widetilde{\eta}, r, r^{\prime}\right)$. Hence, $r^{\prime}$ weakly dominates $r$. Since (i) and (ii) contradict the assumption that $r$ is weakly undominated, $r \in B_{K}^{U}(\eta, \widetilde{\eta})$.

Given the presumed optimal trading strategy, the value function is a step function. $\widehat{V_{K}}(\eta)=$ $V_{K}(\lfloor\eta / p\rfloor)$.

At the conjectured equilibrium, the value function is a step function, continuous from the right, and strictly increasing at integer multiples of $p$. The set of weakly undominated reservation prices is reduced to 


$$
\begin{gathered}
=\left\{\begin{array}{cc}
B_{K}^{I}(\eta) & u+\widehat{V_{K}}(\eta-r) \geq \widehat{V_{K}}(\eta) \wedge \\
r \in[0, \eta] \mid & \forall r^{\prime}>r: u+\widehat{V_{K}}\left(\eta-r^{\prime}\right) \leq \widehat{V_{K}}(\eta)
\end{array}\right\} \\
=\left\{\begin{array}{c}
r \in[0, \eta] \mid \\
u \mu(\widetilde{\eta})+\widehat{V_{K}}(\eta-r)-\widehat{V_{K}}(\eta) \geq 0 \wedge \\
\forall r^{\prime}>r: u \mu(\widetilde{\eta})+\widehat{V_{K}}\left(\eta-r^{\prime}\right)-\widehat{V_{K}}(\eta) \leq 0
\end{array}\right\}
\end{gathered}
$$

The optimal reservation prices are

$$
\begin{aligned}
& \rho_{K}^{I}(\eta)=\max B_{K}^{I}(\eta)=\max \left\{r \in[0, \eta] \mid u+\widehat{V_{K}}(\eta-r) \geq \widehat{V_{K}}(\eta)\right\}, \\
& \rho_{K}^{U}(\eta, \widetilde{\eta})=\max B_{K}^{U}(\eta, \widetilde{\eta})=\max \left\{r \in[0, \eta] \mid u \mu(\widetilde{\eta})+\widehat{V_{K}}(\eta-r)-\widehat{V_{K}}(\eta) \geq 0\right\} . \\
& A_{K}(\eta)= \\
& \left\{\begin{array}{c}
o \in \Re_{+}^{*} \mid\left[o \in \Re_{+} \wedge \widehat{V_{K}}(\eta+o)-\gamma>\widehat{V_{K}}(\eta) \wedge o / p \text { is an integer }\right] \vee \\
{\left[o=\infty \wedge \forall o^{\prime} \in \Re_{+}: \widehat{V_{K}}\left(\eta+o^{\prime}\right)-\gamma \leq \widehat{V_{K}}(\eta)\right]}
\end{array}\right\}
\end{aligned}
$$

The optimal offer is

$$
\begin{aligned}
& \omega_{K}(\eta) \in \arg \max _{o \in A_{K}(\eta)}\left\{\left[1-R^{U}(o, \eta)-R^{I}(o)\right]\left[\widehat{V_{K}}(\eta+o)-\gamma-\widehat{V_{K}}(\eta)\right]\right\} \\
& R^{U}(x, \widetilde{\eta})=G_{H}\left\{\eta \mid \rho_{H}^{U}(\eta, \widetilde{\eta})<x\right\}+G_{L}\left\{\eta \mid \rho_{L}^{U}(\eta, \widetilde{\eta})<x\right\} \\
& R^{I}(x)=G_{H}\left\{\eta \mid \rho_{H}^{I}(\eta)<x\right\}+G_{L}\left\{\eta \mid \rho_{L}^{I}(\eta)<x\right\} .
\end{aligned}
$$

To Summarize:

LEMMA 3. At the wealth-signaling and non-wealth-signaling equilibria, the optimal reservationprice and offer strategies are given by $\rho_{K}^{I}(\eta), \rho_{K}^{U}(\eta, \widetilde{\eta})$ and $\omega_{K}(\eta)$.

The presumed strategy (ix) assumes that the uninformed buyers' belief is 


$$
\mu(\eta)=\left\{\begin{array}{ccc}
\frac{d \Psi_{H}(\eta)}{d \Psi(\eta)} & \text { for } & d \Psi(\eta)>0 \\
0 & \text { for } & d \Psi(\eta)=0
\end{array}\right.
$$

where $\Psi_{H}(x)=G_{H}\{\eta \mid \eta \leq x\}$ and $\Psi(x)=G_{H}\{\eta \mid \eta \leq x\}+G_{L}\{\eta \mid \eta \leq x\}$.

By the presumed optimal strategies (iii) and (iv), a seller offers to sell at price $p$ in state $M_{K}-1$ and is not willing to offer $p$ in state $M_{K}$. By the optimal offer strategy $\omega_{K}(\eta)$,

$$
\begin{aligned}
& V_{K}\left(M_{K}\right)-\gamma>V_{K}\left(M_{K}-1\right) \\
& V_{K}\left(M_{K}+1\right)-\gamma \leq V_{K}\left(M_{K}\right),
\end{aligned}
$$

given the reservation-price distribution.

$R_{K}$ is defined as: Above $R_{K}$ type $K$ buyers who know trading partners' type accept the offer $Q_{H} p$ (made by sellers in state $M_{H}$ ) and below $R_{K}$ buyers reject the offer $Q_{H} p . S_{K}$ is defined as: Above $S_{K}$ type $K$ buyers who do not know trading partners' type accept the offer $Q_{H} p$ (made by both $\mathrm{H}$ and $\mathrm{L}$ sellers in state $M_{H}$ ) and below $S_{K}$ these buyers reject the offer $Q_{H} p$ when $M_{H}=M_{L}$ and $Q_{H}=Q_{L}$. That is:

For any $m \leq R_{K}, u+V_{K}\left(m-Q_{H}\right)<V_{K}(m)$ and $u+V_{K}\left(R_{K}+1-Q_{H}\right) \geq V_{K}\left(R_{K}+1\right)$. Thus,

$$
R_{K}=\max \left\{m \mid u+V_{K}\left(m-Q_{H}\right)<V_{K}(m)\right\} .
$$

For any $R_{K}<m \leq S_{K}, u \frac{d \Psi^{H}\left(M_{H} p\right)}{d \Psi\left(M_{H} p\right)}+V_{K}\left(m-Q_{H}\right)<V_{K}(m)$ and $u \frac{d \Psi^{H}\left(M_{H} p\right)}{d \Psi\left(M_{H} p\right)}+V_{K}\left(S_{K}+\right.$ $\left.1-Q_{H}\right) \geq V_{K}\left(S_{K}+1\right)$. Thus,

$$
S_{K}=\max \left\{m \mid u \frac{d \Psi^{H}\left(M_{H} p\right)}{d \Psi\left(M_{H} p\right)}+V_{K}\left(m-Q_{H}\right)<V_{K}(m)\right\} .
$$

Notice $\frac{d \Psi^{H}(m p)}{d \Psi(m p)}=\mu_{m}$.

The following lemma shows that the part of the uninformed and informed buyers' strategies of the wealth-signaling equilibrium (presumed strategies (i) and (ii)) are optimal.

LEMMA 4. At the first example of the wealth-signaling equilibrium where $M_{K}=Q_{K}=2$ (for $K=H, L)$, for a given set of the parameters, $\bar{M}, u, \gamma, \alpha, r$ and $\theta$, if $V_{K}(2)-V_{K}(1) \leq \mu_{0} u<$ $V_{K}(1)-V_{K}(0) \leq \mu_{1} u(K=H, L)$ (where $\left.\mu_{m}=\frac{P_{m}^{H}}{P_{m}^{H}+P_{m}^{L}}\right)$, then, $\rho_{K}^{I}(m p) \geq p$ for $m=1,2$, 
and $\rho_{K}^{U}(m p, \widetilde{m} p) \geq p$ if $(m, \widetilde{m})=(1,1)$ or $(m=2$ and $\widetilde{m}=0,1)$, and $\rho_{K}^{U}(m p, \widetilde{m} p)<p$ if $(m, \widetilde{m})=(1,0)$.

Proof. For informed agents: Since $V_{K}$ is weakly increasing and $u+V_{K}(m-1) \geq V_{K}(m)$ for $m=1,2, \rho_{K}^{I}(m p) \geq p$ for $m=1,2$.

For uninformed agents: Since $\frac{d \Psi^{H}(m p)}{d \Psi(m p)}=\mu_{m}, \mu_{0} u+V_{K}(1)-V_{K}(2) \geq 0, \mu_{1} u+V_{K}(0)-V_{K}(1) \geq$ $0, \mu_{1} u+V_{K}(1)-V_{K}(2) \geq 0, \mu_{0} u+V_{K}(0)-V_{K}(1)<0$, and $V_{K}$ is weakly increasing, $\rho_{K}^{U}(2 p, 0) \geq p$, $\rho_{K}^{U}(p, p) \geq p, \rho_{K}^{U}(2 p, p) \geq p, \rho_{K}^{U}(p, 0)<p$.

Similarly, the next lemma shows that the informed and uninformed buyers' strategies of the second example of the wealth-signaling and non-wealth-signaling equilibria are optimal.

LEMMA 5. for a given set of the parameters, $\bar{M}, u, \gamma, \alpha, r$ and $\theta$,

(1) at the second example of the wealth-signaling equilibrium where $M_{K}=Q_{K}=2$, if $\mu_{0} u<V_{K}(1)-V_{K}(0) \leq \mu_{1} u$ and $\mu_{0} u<V_{K}(2)-V_{K}(1) \leq \mu_{1} u$, then $\rho_{K}^{I}(m p) \geq p$ for $m=1,2$, and $\rho_{K}^{U}(m p, \widetilde{m} p) \geq p$ if $\widetilde{m}=1$ and $m=1,2$, and $\rho_{K}^{U}(m p, \widetilde{m} p)<p$ if $\widetilde{m}=0$ and $m=1,2$.

(2) at the non-wealth-signaling equilibrium where $M_{K}=Q_{K}=2$, if $V_{K}(2)-V_{K}(1) \leq$ $\min \left(\mu_{0} u, \mu_{1} u\right)$ and $V_{K}(1)-V_{K}(0) \leq \min \left(\mu_{0} u, \mu_{1} u\right)$, then $\rho_{K}^{I}(m p) \geq p$ for $m=1,2$, and $\rho_{K}^{U}(m p, \widetilde{m} p) \geq p$ for $m=1,2$ and $\widetilde{m}=0,1$.

A belief $\mu(\eta)=0$ for out-of-equilibrium money holdings $\eta$ implies the following.

LEMMA 6. $\rho_{K}^{U}(\eta, \widetilde{\eta})<p$ if $\widetilde{\eta} / p$ is not an integer, and $\rho_{K}^{U}(m p, \widetilde{m} p)<p$ for $\widetilde{m} \geq 3$.

In the wealth-signaling and non-wealth-signaling equilibria where $M_{K}=Q_{K}=2$, sellers with two units of money offer price higher than one, i.e., two. However, no buyers with two units of money accept such an offer. Thus:

LEMMA 7. In the (wealth-signaling and non-wealth-signaling) equilibria where $M_{K}=$ $Q_{K}=2$, if $V_{K}(4)-V_{K}(2)>\gamma$, then $V_{K}(2)-V_{K}(0)>u$.

Proof. Suppose not. Then, $V_{K}(2)-V_{K}(0) \leq u$.Thus, the informed buyers buy at $2 p$ from the high types. Trade occurs at $p$ and $2 p$, which contradicts the definition of single-price 
equilibrium.

Hereafter, we will prove the rest of the conditions for existence of the equilibria separately. First, we will prove the conditions for the first example of the wealth-signaling and the nonwealth-signaling equilibria where $M_{K}=Q_{K}=2$. Second, we will prove the rest of the conditions for existence of the second example of the wealth-signaling equilibrium where $M_{K}=Q_{K}=2$.

(I) The first example of the wealth-signaling and non-wealth-signaling equilibria where $M_{K}=Q_{K}=2$.

LEMMA 8. At the first example of the wealth-signaling and the non wealth-signaling equilibria where $M_{K}=Q_{K}=2$, the value function $V$ is strictly concave in $m \in\left[2, R_{K}\right]$.

Proof. For $2 \leq m \leq R_{K}-1$, by equation (9),

$$
\begin{aligned}
& {\left[r / \alpha+(1-\theta) \sum_{\tilde{m}=0}^{1}\left(P_{\tilde{m}}^{H}+P_{\tilde{m}}^{L}\right)+\theta \sum_{\tilde{m}=0}^{1} P_{\tilde{m}}^{H}\right]\left[V_{K}(m+1)-V_{K}(m)\right] } \\
= & {\left[(1-\theta) \sum_{\tilde{m}=0}^{1}\left(P_{\tilde{m}}^{H}+P_{\tilde{m}}^{L}\right)+\theta \sum_{\tilde{m}=0}^{1} P_{\tilde{m}}^{H}\right]\left[V_{K}(m)-V_{K}(m-1)\right] . }
\end{aligned}
$$

Hence, $V_{K}(m+1)-V_{K}(m)<V_{K}(m)-V_{K}(m-1)$ for $m \in\left[2, R_{K}\right)$.

The presumed strategy of the informed buyers with more than $2\left(=M_{K}\right)$ units of money is (i). We presumed the strategy (ii) and (ii)" that the uninformed buyers with more than $2\left(=M_{K}\right)$ units of money accept offer $p$ if the uninformed buyers with 2 units of money accept offer $p$.

LEMMA 9. At the first example of the wealth-signaling and the non-wealth-signaling equilibria,

(a) it is optimal for the informed buyers with more than 2 units of money to accept offer $p$ made by type $H$ sellers.

(b) Suppose that the uninformed buyers with 2 units of money accept offer $p$ made by the sellers with $\widetilde{m}(\in\{0,1\})$ units of money (i.e., $\left.\rho_{K}^{U}(2 p, \widetilde{m} p) \geq p\right)$ and 
$\max \left\{\theta P_{2}^{H}\left(P_{\tilde{m}}^{L}-P_{\tilde{m}}^{H}\right) / P_{\tilde{m}}^{H},(1-\theta)\left[P_{2}^{H}\left(P_{\tilde{m}}^{L} / P_{\tilde{m}}^{H}\right)-P_{2}^{L}\right]\right\} \leq r / \alpha$ for $\widetilde{m}$. Then, it is optimal for the uninformed buyers with more than 2 units of money to accept offer $p$ made by the sellers with $\widetilde{m}$ units of money (i.e., $\rho_{K}^{U}(m p, \widetilde{m} p) \geq p$ for $m>2$.).

Proof. (a) The informed buyers:

For $m \in\left[2, R_{K}\right)$, since $V_{K}(m+1)-V_{K}(m)<V_{K}(m)-V_{K}(m-1)$ and $V_{K}(2)-V_{K}(1) \leq u$, $V_{K}(m+1)-V_{K}(m) \leq u$.

For $m=R_{K}$, since $V_{K}\left(R_{K}+1\right)-V_{K}\left(R_{K}-1\right) \leq u$ (by definition of $R_{K}$ ) and $V_{K}$ is an increasing function, $V_{K}\left(R_{K}+1\right)-V_{K}\left(R_{K}\right) \leq u$.

For $R_{K}+1 \leq m \leq S_{K}-1$, by equation (10),

$$
\begin{aligned}
& {\left[r / \alpha+(1-\theta) \sum_{\tilde{m}=0}^{1}\left(P_{\tilde{m}}^{H}+P_{\tilde{m}}^{L}\right)+\theta \sum_{\tilde{m}=0}^{1} P_{\tilde{m}}^{H}+\theta P_{2}^{H}\right]\left[V_{K}(m+1)-V_{K}(m)\right] } \\
= & {\left[(1-\theta) \sum_{\tilde{m}=0}^{1}\left(P_{\tilde{m}}^{H}+P_{\tilde{m}}^{L}\right)+\theta \sum_{\tilde{m}=0}^{1} P_{\tilde{m}}^{H}\right]\left[V_{K}(m)-V_{K}(m-1)\right] } \\
& +\theta P_{2}^{H}\left[V_{K}(m-1)-V_{K}(m-2)\right]
\end{aligned}
$$

Since $V_{K}\left(R_{K}+1\right)-V_{K}\left(R_{K}\right) \leq u$ and $V_{K}\left(R_{K}\right)-V_{K}\left(R_{K}-1\right) \leq u$ by the concavity of $V_{K}$ up to $R_{K}$. Thus, applying the above equation recursively, $V_{K}(m+1)-V_{K}(m) \leq u$ for $R_{K}+1 \leq m \leq S_{K}-1$.

For $m=S_{K}$, since $V_{K}\left(S_{K}+1\right)-V_{K}\left(S_{K}-1\right) \leq \mu_{2} u \leq u$ (by definition of $S_{K}$ ) and $V_{K}$ is an increasing function, $V_{K}\left(S_{K}+1\right)-V_{K}\left(S_{K}\right) \leq u$.

For $m \geq S_{K}+1$, by equation (11),

$$
\begin{aligned}
& {\left[r / \alpha+(1-\theta) \sum_{\tilde{m}=0}^{1}\left(P_{\tilde{m}}^{H}+P_{\tilde{m}}^{L}\right)+\theta \sum_{\tilde{m}=0}^{1} P_{\tilde{m}}^{H}+P_{2}^{H}+(1-\theta) P_{2}^{L}\right] } \\
& {\left[V_{K}(m+1)-V_{K}(m)\right] } \\
= & {\left[(1-\theta) \sum_{\tilde{m}=0}^{1}\left(P_{\tilde{m}}^{H}+P_{\tilde{m}}^{L}\right)+\theta \sum_{\tilde{m}=0}^{1} P_{\tilde{m}}^{H}\right]\left[V_{K}(m)-V_{K}(m-1)\right] } \\
& +\left[P_{2}^{H}+(1-\theta) P_{2}^{L}\right]\left[V_{K}(m-1)-V_{K}(m-2)\right]
\end{aligned}
$$


Since $V_{K}\left(S_{K}+1\right)-V_{K}\left(S_{K}\right) \leq u$ and $V_{K}\left(S_{K}\right)-V_{K}\left(S_{K}-1\right) \leq u$ if $S_{K}>R_{K}$ from above. Thus, applying the above equation recursively, $V_{K}(m+1)-V_{K}(m) \leq u$ for $m \geq S_{K}+1$.

(b) The uninformed buyers:

For $m \in\left[2, R_{K}\right)$, since $V_{K}(m+1)-V_{K}(m)<V_{K}(m)-V_{K}(m-1)$, if $V_{K}(2)-V_{K}(1) \leq \mu_{\tilde{m}} u$ for $\widetilde{m} \in\{0,1\}, V_{K}(m+1)-V_{K}(m) \leq \mu_{\tilde{m}} u$.

For $m=R_{K}$, by equations (9) and (10),

$$
\begin{aligned}
& {\left[r / \alpha+(1-\theta) \sum_{\tilde{m}=0}^{1}\left(P_{\tilde{m}}^{H}+P_{\tilde{m}}^{L}\right)+\theta \sum_{\tilde{m}=0}^{1} P_{\tilde{m}}^{H}+\theta P_{2}^{H}\right]\left[V_{K}\left(R_{K}+1\right)-V_{K}\left(R_{K}\right)\right] } \\
= & {\left[(1-\theta) \sum_{\tilde{m}=0}^{1}\left(P_{\tilde{m}}^{H}+P_{\tilde{m}}^{L}\right)+\theta \sum_{\tilde{m}=0}^{1} P_{\tilde{m}}^{H}-\theta P_{2}^{H}\right]\left[V_{K}\left(R_{K}\right)-V_{K}\left(R_{K}-1\right)\right]+\theta P_{2}^{H} u }
\end{aligned}
$$

Since $V_{K}\left(R_{K}\right)-V_{K}\left(R_{K}-1\right) \leq \mu_{\tilde{m}} u$, if $\theta P_{2}^{H}\left(P_{\tilde{m}}^{L}-P_{\tilde{m}}^{H}\right) / P_{\tilde{m}}^{H} \leq r / \alpha$, then $V_{K}\left(R_{K}+1\right)-$ $V_{K}\left(R_{K}\right) \leq \mu_{\tilde{m}} u$ for $\tilde{m} \in\{0,1\}$.

For $R_{K}+1 \leq m \leq S_{K}-1$, by equation (10), equation (A1) holds. Since $V_{K}\left(R_{K}+1\right)-$ $V_{K}\left(R_{K}\right) \leq \mu_{\tilde{m}} u$ and $V_{K}\left(R_{K}\right)-V_{K}\left(R_{K}-1\right) \leq \mu_{\tilde{m}} u, V_{K}(m+1)-V_{K}(m) \leq \mu_{\tilde{m}} u$.

For $m=S_{K}$, by equation (10),

$$
\begin{aligned}
& {\left[r / \alpha+(1-\theta) \sum_{\tilde{m}=0}^{1}\left(P_{\tilde{m}}^{H}+P_{\tilde{m}}^{L}\right)+\theta \sum_{\tilde{m}=0}^{1} P_{\tilde{m}}^{H}+\theta P_{2}^{H}+(1-\theta)\left(P_{2}^{H}+P_{2}^{L}\right)\right] } \\
& {\left[V_{K}\left(S_{K}+1\right)-V_{K}\left(S_{K}\right)\right] } \\
= & {\left[(1-\theta) \sum_{\tilde{m}=0}^{1}\left(P_{\tilde{m}}^{H}+P_{\tilde{m}}^{L}\right)+\theta \sum_{\tilde{m}=0}^{1} P_{\tilde{m}}^{H}\right]\left[V_{K}\left(S_{K}\right)-V_{K}\left(S_{K}-1\right)\right] } \\
& +\theta P_{2}^{H}\left[V_{K}\left(S_{K}-1\right)-V_{K}\left(S_{K}-2\right)\right]+(1-\theta) P_{2}^{H} u
\end{aligned}
$$

Since $V_{K}\left(S_{K}\right)-V_{K}\left(S_{K}-1\right) \leq \mu_{\tilde{m}} u$ and $V_{K}\left(S_{K}-1\right)-V_{K}\left(S_{K}-2\right) \leq \mu_{\tilde{m}} u$, if $(1-\theta)\left[P_{2}^{H}\left(P_{\tilde{m}}^{L} / P_{\tilde{m}}^{H}\right)-P_{2}^{L}\right] \leq r / \alpha$, then $V_{K}\left(S_{K}+1\right)-V_{K}\left(S_{K}\right) \leq \mu_{\widetilde{m}} u$.

For $m \geq S_{K}+1$, by equation (11), equation (A2) holds. Since $V_{K}\left(S_{K}\right)-V_{K}\left(S_{K}-1\right) \leq \mu_{\tilde{m}} u$ and $V_{K}\left(S_{K}+1\right)-V_{K}\left(S_{K}\right) \leq \mu_{\tilde{m}} u\left(\right.$ if $\theta P_{2}^{H}\left(P_{\tilde{m}}^{L}-P_{\tilde{m}}^{H}\right) / P_{\tilde{m}}^{H} \leq r / \alpha$ and $(1-\theta)\left[P_{2}^{H}\left(P_{\tilde{m}}^{L} / P_{\tilde{m}}^{H}\right)-P_{2}^{L}\right] \leq$ 
$r / \alpha), V_{K}\left(S_{K}+2\right)-V_{K}\left(S_{K}+1\right) \leq \mu_{\tilde{m}} u$. Applying equation (A2) recursively, $V_{K}(m+1)-V_{K}(m) \leq$ $\mu_{\tilde{m}} u$ for $m \geq S_{K}+1$ if $\theta P_{2}^{H}\left(P_{\tilde{m}}^{L}-P_{\tilde{m}}^{H}\right) / P_{\tilde{m}}^{H} \leq r / \alpha$ and $(1-\theta)\left[P_{2}^{H}\left(P_{\tilde{m}}^{L} / P_{\tilde{m}}^{H}\right)-P_{2}^{L}\right] \leq r / \alpha$.

LEMMA 10. Suppose that $r / \alpha \geq \theta P_{2}^{L}$, and if $V_{K}(4)-V_{K}(2)>\gamma$, then $V_{K}(2)-V_{K}(0)>u$.

Then, at the first example of the wealth-signaling and the non-wealth-signaling equilibria, it is optimal for buyers

(a) in state up to 2 (in the support) to reject offer $2 p$ or higher,

(b) in state up to $R_{K}$ to reject offer $2 p$,

(c) who know a trading partner's type in state above $R_{K}$ to accept offer $2 p$ from the high type,

(d) who do not know a trading partner's type in state above $S_{K}$ to accept offer $2 p$ made by sellers in state of 2 but in state up to $S_{K}$ to reject offer $2 p$.

Proof. (a) Since $V_{K}(2)-V_{K}(0)>u$, the informed and uninformed buyers with 2 units of money reject offer $2 p$.

(b) is shown by $R_{K}=\max \left\{m \mid u+V_{K}(m-2)<V_{K}(m)\right\}$, which implies that for any $n \leq$ $R_{K}, u+V_{K}(n-2)<V_{K}(n)$.

(c) and (d) To show (c) and (d), show that for $R_{K}+1 \leq m \leq S_{K}, \mu_{2} u<V_{K}(m)-V_{K}(m-2) \leq$ $u$ and for $S_{K}+1 \leq m, V_{K}(m)-V_{K}(m-2) \leq \mu_{2} u$. (If $R_{K}=S_{K}$, we need to show only, for $S_{K}+1 \leq m, V_{K}(m)-V_{K}(m-2) \leq \mu_{2} u$.)

Obviously, $R_{K} \leq S_{K}$. By the definitions of $R_{K}$ and $S_{K}, V_{K}\left(R_{K}+1\right)-V_{K}\left(R_{K}-1\right) \leq u$, $V_{K}\left(S_{K}+1\right)-V_{K}\left(S_{K}-1\right) \leq \mu_{2} u$ and for $R_{K}+1 \leq m \leq S_{K}, V_{K}(m)-V_{K}(m-2)>\mu_{2} u$.

If $R_{K}+2 \leq S_{K}$, consider the following four cases: (1) $R_{K}+1 \leq m \leq R_{K}+2$, (2) $R_{K}+3 \leq$ $m \leq S_{K}$ (if $R_{K}+2=S_{K},(2)$ is $\left.\emptyset\right),(3) S_{K}+1 \leq m \leq S_{K}+2$, and (4) $S_{K}+3 \leq m$. If $R_{K}+2>S_{K}$, consider the following four cases: (1)' $R_{K}+1 \leq m \leq S_{K}$, (2)' $S_{K}+1 \leq m \leq R_{K}+2$, (3)' $R_{K}+3 \leq m \leq S_{K}+2,(4)^{\prime} S_{K}+3 \leq m$. 
Case (1). $V_{K}\left(R_{K}+1\right)-V_{K}\left(R_{K}-1\right) \leq u$ by the definition of $R_{K}$. By equations (9) and (10), for $m=R_{K}+2$,

$$
\begin{aligned}
& {\left[r / \alpha+(1-\theta) \sum_{\tilde{m}=0}^{1}\left(P_{\tilde{m}}^{H}+P_{\tilde{m}}^{L}\right)+\theta \sum_{\tilde{m}=0}^{1} P_{\tilde{m}}^{H}+\theta P_{2}^{H}\right]\left[V_{K}(m)-V_{K}(m-2)\right] } \\
= & {\left[(1-\theta) \sum_{\tilde{m}=0}^{1}\left(P_{\tilde{m}}^{H}+P_{\tilde{m}}^{L}\right)+\theta \sum_{\tilde{m}=0}^{1} P_{\tilde{m}}^{H}\right]\left[V_{K}(m-1)-V_{K}(m-3)\right]+\theta P_{2}^{H} u . }
\end{aligned}
$$

Thus, $V_{K}(m)-V_{K}(m-2) \leq u$. Hence, $V_{K}(m)-V_{K}(m-2) \leq u$ for $R_{K}+1 \leq m \leq R_{K}+2$. Case (2). By equation (10),

$$
\begin{aligned}
& {\left[r / \alpha+(1-\theta) \sum_{\tilde{m}=0}^{1}\left(P_{\tilde{m}}^{H}+P_{\tilde{m}}^{L}\right)+\theta \sum_{\tilde{m}=0}^{1} P_{\tilde{m}}^{H}+\theta P_{2}^{H}\right]\left[V_{K}(m)-V_{K}(m-2)\right] } \\
= & {\left[(1-\theta) \sum_{\tilde{m}=0}^{1}\left(P_{\tilde{m}}^{H}+P_{\tilde{m}}^{L}\right)+\theta \sum_{\tilde{m}=0}^{1} P_{\tilde{m}}^{H}\right]\left[V_{K}(m-1)-V_{K}(m-3)\right] } \\
& +\theta P_{2}^{H}\left[V_{K}(m-2)-V_{K}(m-4)\right] .
\end{aligned}
$$

Since $V_{K}\left(R_{K}+1\right)-V_{K}\left(R_{K}-1\right) \leq u$ and $V_{K}\left(R_{K}+2\right)-V_{K}\left(R_{K}\right) \leq u$ (by the proof of case (1)), $V_{K}\left(R_{K}+3\right)-V_{K}\left(R_{K}+1\right) \leq u$. It implies $V_{K}(m)-V_{K}(m-2) \leq u$ for $R_{K}+3 \leq m \leq S_{K}$ by applying the above equation recursively.

Case (3). By equations (10) and (11),

$$
\begin{aligned}
& {\left[r / \alpha+(1-\theta) \sum_{\tilde{m}=0}^{1}\left(P_{\tilde{m}}^{H}+P_{\tilde{m}}^{L}\right)+\theta \sum_{\tilde{m}=0}^{1} P_{\tilde{m}}^{H}+\theta P_{2}^{H}+(1-\theta)\left(P_{2}^{H}+P_{2}^{L}\right)\right] } \\
& {\left[V_{K}(m)-V_{K}(m-2)\right] } \\
= & {\left[(1-\theta) \sum_{\tilde{m}=0}^{1}\left(P_{\tilde{m}}^{H}+P_{\tilde{m}}^{L}\right)+\theta \sum_{\tilde{m}=0}^{1} P_{\tilde{m}}^{H}\right]\left[V_{K}(m-1)-V_{K}(m-3)\right] } \\
& +\theta P_{2}^{H}\left[V_{K}(m-2)-V_{K}(m-4)\right]+(1-\theta) P_{2}^{H} u .
\end{aligned}
$$


Since $V_{K}\left(S_{K}+1\right)-V_{K}\left(S_{K}-1\right) \leq \mu_{2} u$ (by the definition of $\left.S_{K}\right)$ and $V_{K}\left(S_{K}\right)-V_{K}\left(S_{K}-2\right) \leq$ $u$ (by the proof of case (2)), if $r / \alpha \geq \theta P_{2}^{L}, V_{K}(m)-V_{K}(m-2) \leq \mu_{2} u$ for $m=S_{K}+2$ by the above equation.

Case (4). By equation (11),

$$
\begin{aligned}
& {\left[r / \alpha+(1-\theta) \sum_{\tilde{m}=0}^{1}\left(P_{\tilde{m}}^{H}+P_{\tilde{m}}^{L}\right)+\theta \sum_{\tilde{m}=0}^{1} P_{\tilde{m}}^{H}+\theta P_{2}^{H}+(1-\theta)\left(P_{2}^{H}+P_{2}^{L}\right)\right] } \\
& {\left[V_{K}(m)-V_{K}(m-2)\right] } \\
= & {\left[(1-\theta) \sum_{\tilde{m}=0}^{1}\left(P_{\tilde{m}}^{H}+P_{\tilde{m}}^{L}\right)+\theta \sum_{\tilde{m}=0}^{1} P_{\tilde{m}}^{H}\right]\left[V_{K}(m-1)-V_{K}(m-3)\right] } \\
& +\left[\theta P_{2}^{H}+(1-\theta)\left(P_{2}^{H}+P_{2}^{L}\right)\right]\left[V_{K}(m-2)-V_{K}(m-4)\right] .
\end{aligned}
$$

Since $V_{K}\left(S_{K}+1\right)-V_{K}\left(S_{K}-1\right) \leq \mu_{2} u$ and $V_{K}\left(S_{K}+2\right)-V_{K}\left(S_{K}\right) \leq \mu_{2} u$ if $r / \alpha \geq \theta P_{2}^{L}$ (by the proof of case (3)), $V_{K}\left(S_{K}+3\right)-V_{K}\left(S_{K}+1\right) \leq \mu_{2} u$. It implies $V_{K}(m)-V_{K}(m-2) \leq \mu_{2} u$ for $S_{K}+3 \leq m$ by applying the above equation recursively.

Case (1)' The proof is the same as the proof of case (1).

Case (2)' By equations (9) and (11),

$$
\begin{aligned}
& {\left[r / \alpha+(1-\theta) \sum_{\tilde{m}=0}^{1}\left(P_{\tilde{m}}^{H}+P_{\tilde{m}}^{L}\right)+\theta \sum_{\tilde{m}=0}^{1} P_{\tilde{m}}^{H}+\theta P_{2}^{H}+(1-\theta)\left(P_{2}^{H}+P_{2}^{L}\right)\right] } \\
& {\left[V_{K}(m)-V_{K}(m-2)\right] } \\
= & {\left[(1-\theta) \sum_{\tilde{m}=0}^{1}\left(P_{\tilde{m}}^{H}+P_{\tilde{m}}^{L}\right)+\theta \sum_{\tilde{m}=0}^{1} P_{\tilde{m}}^{H}\right]\left[V_{K}(m-1)-V_{K}(m-3)\right]+P_{2}^{H} u . }
\end{aligned}
$$

Since $V_{K}\left(S_{K}+1\right)-V_{K}\left(S_{K}-1\right) \leq \mu_{2} u$, if $r / \alpha \geq \theta P_{2}^{L}, V_{K}(m)-V_{K}(m-2) \leq \mu_{2} u$ for $S_{K}+1 \leq m \leq R_{K}+2$ by applying the above equation recursively.

Case (3)' The proof is the same as the proof of case (3), except that "(by the proof of case $(2))$ " is replaced with "(by the proof of case (1)')".

Case (4)' The proof is the same as the proof of case (4), except that "(by the proof of case $(3))$ " is replaced with "(by the proof of case $\left.(3)^{\prime}\right)$ ". 
Next, find the conditions that offer strategy profile (iii), (iv) and (vi) are optimal. By $A_{K}(\eta)$, (vi) is weakly undominated if and only if $V_{K}\left(M_{K}+Q_{K}\right)-V_{K}\left(M_{K}\right)>\gamma$, i.e., $V_{K}(4)-V_{K}(2)>\gamma$.

LEMMA 11. Suppose $V_{K}(3)-V_{K}(2) \leq \gamma<V_{K}(2)-V_{K}(1), V_{K}(1)-V_{K}(0)>\gamma$, and if $V_{K}(4)-V_{K}(2)>\gamma$, then $V_{K}(2)-V_{K}(0)>u$.

Then, it is optimal for sellers with money holdings of less than $2 p$ to offer $p$. Furthermore, suppose that if $R_{K}=3$, then $V_{K}(4)-V_{K}(3) \leq \gamma$, then it is optimal for sellers with money holdings of at least $2 p$ to offer a price above $p$.

Proof. (I) Consider a seller with money $m p$ and $m<2$.

By $V_{K}(2)-V_{K}(0)>u$ for all $j>1, R^{I}(j p)+R^{U}(j p, m p)=1$. Since $V_{K}(2)-V_{K}(1)>\gamma$ and $V_{K}(1)-V_{K}(0)>\gamma$, given $R^{I}$ and $R^{U}$, by the net expected value of an agent in state $m$ offering $j p: W(m, j)=\left[1-R^{I}(j p)-R^{U}(j p, m p)\right]\left[V_{K}(m+j)-\gamma-V_{K}(m)\right]$.

For $m=0,1$ and $j>1, W(m, 1)=\left(1-\theta+1_{K} \theta\right) \sum_{\tilde{m}=1}^{2}\left(P_{\tilde{m}}^{H}+P_{\tilde{m}}^{L}\right)\left[V_{K}(m+1)-\gamma-V_{K}(m)\right]$ $>W(m, j)=0>W(m, 0)$, since $V_{K}(2)-V_{K}(1)>\gamma$ and $V_{K}(1)-V_{K}(0)>\gamma \cdot\left(1_{K}=1\right.$ if $K=H$, and 0 if $K=L)$. Thus, for $m=0,1, \omega(m p)=p$, That is, all agents holding less than $2 p$ units of money should offer exactly $p$.

(II) Next, consider an agent with money $m p$ and $m \geq 2$. To show $\omega(m p)>p$, it is sufficient to show $p \notin A_{K}(\eta)$, or $V_{K}(m+1)-V_{K}(m) \leq \gamma$.

(i) For $m \in\left[2, R_{K}\right)$ : By lemma showing concavity of $V_{K}$ for $m \leq R_{K}$ and $V_{K}(3)-V_{K}(2) \leq \gamma$, for any $m \in\left[2, R_{K}\right), V_{K}(m+1)-V_{K}(m) \leq \gamma$.

(ii) For $m \in\left[R_{K}, S_{K}\right)$ :

(1) Consider the case that $R_{K}>3$. By the definition of $R_{K}, V_{K}\left(R_{K}+1\right)-V_{K}\left(R_{K}-1\right) \leq$ $u<V_{K}\left(R_{K}\right)-V_{K}\left(R_{K}-2\right)$; thus, $V_{K}\left(R_{K}+1\right)-V_{K}\left(R_{K}\right)<V_{K}\left(R_{K}-1\right)-V_{K}\left(R_{K}-2\right) \leq \gamma$, since $R_{K}-2 \in\left[2, R_{K}\right)$. 
For $m \in\left[R_{K}+1, S_{K}\right)$, by equation (10),

$$
\begin{aligned}
& {\left[r / \alpha+(1-\theta) \sum_{\tilde{m}=0}^{1}\left(P_{\tilde{m}}^{H}+P_{\tilde{m}}^{L}\right)+\theta \sum_{\tilde{m}=0}^{1} P_{\tilde{m}}^{H}+\theta P_{2}^{H}\right]\left[V_{K}(m+1)-V_{K}(m)\right] } \\
= & {\left[(1-\theta) \sum_{\tilde{m}=0}^{1}\left(P_{\tilde{m}}^{H}+P_{\tilde{m}}^{L}\right)+\theta \sum_{\tilde{m}=0}^{1} P_{\tilde{m}}^{H}\right]\left[V_{K}(m)-V_{K}(m-1)\right] } \\
& +\theta P_{2}^{H}\left[V_{K}(m-1)-V_{K}(m-2)\right] .
\end{aligned}
$$

Since $V_{K}\left(R_{K}+1\right)-V_{K}\left(R_{K}\right) \leq \gamma$ and $V_{K}\left(R_{K}\right)-V_{K}\left(R_{K}-1\right) \leq \gamma$, starting with $m=R_{K}+1$, and applying equation (A3) recursively, $V_{K}(m+1)-V_{K}(m) \leq \gamma$ for $m \in\left[R_{K}+1, S_{K}\right)$. Hence, $V_{K}(m+1)-V_{K}(m) \leq \gamma$ for $m \in\left[R_{K}, S_{K}\right)$.

(2) Consider the case that $R_{K}=3$. By using the assumption that $V_{K}(4)-V_{K}(3) \leq \gamma$ and $V_{K}(3)-V_{K}(2) \leq \gamma$, and applying equation (A3) recursively, $V_{K}(m+1)-V_{K}(m) \leq \gamma$ for $m \in\left[R_{K}+1, S_{K}\right)$. Hence, $V_{K}(m+1)-V_{K}(m) \leq \gamma$ for $m \in\left[R_{K}, S_{K}\right)$.

(iii) For $m \geq S_{K}+1$ :

(1) Consider the case that $S_{K}>3$. By the definition of $S_{K}, V_{K}\left(S_{K}+1\right)-V_{K}\left(S_{K}-1\right) \leq$ $\mu_{2} u<V_{K}\left(S_{K}\right)-V_{K}\left(S_{K}-2\right)$; thus, $V_{K}\left(S_{K}+1\right)-V_{K}\left(S_{K}\right)<V_{K}\left(S_{K}-1\right)-V_{K}\left(S_{K}-2\right) \leq \gamma$, since $S_{K}-2 \in\left[2, S_{K}\right)$.

For $m \geq S_{K}+1$, by equation (11),

$$
\begin{aligned}
& {\left[r / \alpha+(1-\theta) \sum_{\tilde{m}=0}^{1}\left(P_{\tilde{m}}^{H}+P_{\tilde{m}}^{L}\right)+\theta \sum_{\tilde{m}=0}^{1} P_{\tilde{m}}^{H}+\theta P_{2}^{H}+(1-\theta)\left(P_{2}^{H}+P_{2}^{L}\right)\right] } \\
& {\left[V_{K}(m+1)-V_{K}(m)\right] } \\
= & {\left[(1-\theta) \sum_{\tilde{m}=0}^{1}\left(P_{\tilde{m}}^{H}+P_{\tilde{m}}^{L}\right)+\theta \sum_{\tilde{m}=0}^{1} P_{\tilde{m}}^{H}\right]\left[V_{K}(m)-V_{K}(m-1)\right] } \\
& +\left[\theta P_{2}^{H}+(1-\theta)\left(P_{2}^{H}+P_{2}^{L}\right)\right]\left[V_{K}(m-1)-V_{K}(m-2)\right] .
\end{aligned}
$$


Since $V_{K}\left(S_{K}+1\right)-V_{K}\left(S_{K}\right) \leq \gamma$ and $V_{K}\left(S_{K}\right)-V_{K}\left(S_{K}-1\right) \leq \gamma$, starting with $m=S_{K}+1$, and applying equation (A4) recursively, $V_{K}(m+1)-V_{K}(m) \leq \gamma$ for $m \geq S_{K}+1$. Hence, $V_{K}(m+1)-V_{K}(m) \leq \gamma$ for $m \geq S_{K}$.

(2) Consider the case that $S_{K}=R_{K}=3$. By using the assumption that $V_{K}(4)-V_{K}(3) \leq \gamma$ and $V_{K}(3)-V_{K}(2) \leq \gamma$, and applying equation (A4) recursively, $V_{K}(m+1)-V_{K}(m) \leq \gamma$ for $m \geq S_{K}+1$. Hence, $V_{K}(m+1)-V_{K}(m) \leq \gamma$ for $m \geq S_{K}$.

In sum, for all $m \geq 2, V_{K}(m+1)-V_{K}(m) \leq \gamma$.

The discussion and conditions up to this point in Appendix is summarized in Lemma1 (a) and $(\mathrm{c})$.

(II) The second example of the wealth-signaling equilibrium where $M_{K}=Q_{K}=2$ (for $K=H, L)$.

$T_{K}$ is defined as: $T_{K}=\max \left\{m \mid \mu_{0} u+V_{K}(m-1)<V_{K}(m)\right\}$. That is, For any $m \leq T_{K}$, $\mu_{0} u+V_{K}(m-1)<V_{K}(m)$ and $\mu_{0} u+V_{K}\left(T_{K}\right) \geq V_{K}\left(T_{K}+1\right)$.

Since $\mu_{2}=1$ from the distribution of money, $R_{K}=S_{K}$.

LEMMA 12. At the second example of the wealth-signaling where $M_{K}=Q_{K}=2$, the value function $V$ is strictly concave in $m \in\left[2, \min \left(T_{K}, R_{K}\right)\right]$.

Proof. For $2 \leq m \leq \min \left(T_{K}, R_{K}\right)-1$, by equation (9),

$$
\begin{aligned}
& {\left[r / \alpha+(1-\theta) P_{1}+\theta \sum_{\tilde{m}=0}^{1} P_{\tilde{m}}^{H}\right]\left[V_{K}(m+1)-V_{K}(m)\right] } \\
= & {\left[(1-\theta) P_{1}+\theta \sum_{\tilde{m}=0}^{1} P_{\tilde{m}}^{H}\right]\left[V_{K}(m)-V_{K}(m-1)\right], }
\end{aligned}
$$

where $P_{m}=P_{m}^{H}+P_{m}^{L}$.

Hence, $V_{K}(m+1)-V_{K}(m)<V_{K}(m)-V_{K}(m-1)$ for $m \in\left[2, \min \left(T_{K}, R_{K}\right)\right)$

LEMMA 13. At the second example of the wealth-signaling equilibria, 
(a) it is optimal for the informed buyers with more than 2 units of money to accept offer $p$ made by type $H$ sellers.

(b) If $\rho_{K}^{U}(2 p, p) \geq p$, then $\rho_{K}^{U}(m p, p) \geq p$ for $m>2$.

(c) Suppose that $P_{2}^{H} P_{0}^{L} / P_{0}^{H} \leq r / \alpha$ when $T_{K}>R_{K}$, and $P_{2}^{H}\left(P_{0}^{L}-P_{0}^{H}\right) / P_{0}^{H} \leq r / \alpha$ when $T_{K} \leq R_{K}$. If $\rho_{K}^{U}(2 p, 0)<p$, then $\rho_{K}^{U}(m p, 0)<p$ for $2<m \leq T_{K}$ and $\rho_{K}^{U}(m p, 0) \geq p$ for $m \geq T_{K}+1$.

Proof. (a) The informed buyers:

For $m \in\left[2, \min \left(T_{K}, R_{K}\right)\right)$, since $V_{K}(m+1)-V_{K}(m)<V_{K}(m)-V_{K}(m-1)$ and $V_{K}(2)-$ $V_{K}(1) \leq u, V_{K}(m+1)-V_{K}(m) \leq u$.

(i) Consider the case that $T_{K}>R_{K}=S_{K}$.

For $m=R_{K}$, since $V_{K}\left(R_{K}+1\right)-V_{K}\left(R_{K}-1\right) \leq u$ (by definition of $R_{K}$ ) and $V_{K}$ is an increasing function, $V_{K}\left(R_{K}+1\right)-V_{K}\left(R_{K}\right) \leq u$.

For $R_{K}+1 \leq m \leq T_{K}-1$, by equation (11),

$$
\begin{aligned}
& {\left[r / \alpha+(1-\theta) P_{1}+\theta \sum_{\tilde{m}=0}^{1} P_{\tilde{m}}^{H}+P_{2}^{H}\right]\left[V_{K}(m+1)-V_{K}(m)\right] } \\
= & {\left[(1-\theta) P_{1}+\theta \sum_{\tilde{m}=0}^{1} P_{\tilde{m}}^{H}\right]\left[V_{K}(m)-V_{K}(m-1)\right]+P_{2}^{H}\left[V_{K}(m-1)-V_{K}(m-2)\right] }
\end{aligned}
$$

Since $V_{K}\left(R_{K}+1\right)-V_{K}\left(R_{K}\right) \leq u$ and $V_{K}\left(R_{K}\right)-V_{K}\left(R_{K}-1\right) \leq u$ by the concavity of $V_{K}$ up to $R_{K}$. Thus, applying the above equation recursively, $V_{K}(m+1)-V_{K}(m) \leq u$ for $R_{K}+1 \leq m \leq T_{K}-1$.

For $m=T_{K}$,

$$
\begin{aligned}
& {\left[r / \alpha+(1-\theta) P_{1}+\theta \sum_{\tilde{m}=0}^{1} P_{\tilde{m}}^{H}+P_{2}^{H}+(1-\theta) P_{0}\right]\left[V_{K}(m+1)-V_{K}(m)\right] } \\
= & {\left[(1-\theta) P_{1}+\theta \sum_{\tilde{m}=0}^{1} P_{\tilde{m}}^{H}\right]\left[V_{K}(m)-V_{K}(m-1)\right] } \\
& +P_{2}^{H}\left[V_{K}(m-1)-V_{K}(m-2)\right]+(1-\theta) P_{0} \mu_{0} u
\end{aligned}
$$


Since $V_{K}\left(T_{K}\right)-V_{K}\left(T_{K}-1\right) \leq u$ and $V_{K}\left(T_{K}-1\right)-V_{K}\left(T_{K}-2\right) \leq u$ from above, $V_{K}\left(T_{K}+1\right)-$ $V_{K}\left(T_{K}\right) \leq u$.

For $m \geq T_{K}+1$, by equation (11),

$$
\begin{aligned}
& {\left[r / \alpha+(1-\theta) P_{1}+P_{0}+\theta \sum_{\tilde{m}=0}^{1} P_{\tilde{m}}^{H}+P_{2}^{H}\right]\left[V_{K}(m+1)-V_{K}(m)\right] } \\
= & {\left[(1-\theta) P_{1}+P_{0}+\theta \sum_{\tilde{m}=0}^{1} P_{\tilde{m}}^{H}\right]\left[V_{K}(m)-V_{K}(m-1)\right] } \\
& +P_{2}^{H}\left[V_{K}(m-1)-V_{K}(m-2)\right]
\end{aligned}
$$

Since $V_{K}\left(T_{K}+1\right)-V_{K}\left(T_{K}\right) \leq u$ and $V_{K}\left(T_{K}\right)-V_{K}\left(T_{K}-1\right) \leq u$ from above, $V_{K}\left(T_{K}+2\right)-$ $V_{K}\left(T_{K}\right) \leq u$. Thus, applying the above equation recursively, $V_{K}(m+1)-V_{K}(m) \leq u$ for $m \geq T_{K}+1$.

(ii) Consider the case that $T_{K} \leq R_{K}=S_{K}$.

For $m=T_{K}$,

$$
\begin{aligned}
& {\left[r / \alpha+(1-\theta) P_{1}+\theta \sum_{\tilde{m}=0}^{1} P_{\tilde{m}}^{H}+(1-\theta) P_{0}\right]\left[V_{K}(m+1)-V_{K}(m)\right] } \\
= & {\left[(1-\theta) P_{1}+\theta \sum_{\tilde{m}=0}^{1} P_{\tilde{m}}^{H}\right]\left[V_{K}(m)-V_{K}(m-1)\right]+(1-\theta) P_{0} \mu_{0} u }
\end{aligned}
$$

Since $V_{K}\left(T_{K}\right)-V_{K}\left(T_{K}-1\right) \leq u, V_{K}\left(T_{K}+1\right)-V_{K}\left(T_{K}\right) \leq u$.

For $T_{K}+1 \leq m \leq R_{K}$, by equation (9),

$$
\begin{aligned}
& {\left[r / \alpha+(1-\theta) P_{1}+P_{0}+\theta \sum_{\widetilde{m}=0}^{1} P_{\tilde{m}}^{H}\right]\left[V_{K}(m+1)-V_{K}(m)\right] } \\
= & {\left[(1-\theta) P_{1}+P_{0}+\theta \sum_{\tilde{m}=0}^{1} P_{\tilde{m}}^{H}\right]\left[V_{K}(m)-V_{K}(m-1)\right] }
\end{aligned}
$$


Since $V_{K}\left(T_{K}+1\right)-V_{K}\left(T_{K}\right) \leq u$, by applying the above equation recursively, $V_{K}(m+1)-$ $V_{K}(m) \leq u$ for $T_{K}+1 \leq m \leq R_{K}$.

For $m=R_{K}$,

$$
\begin{aligned}
& {\left[r / \alpha+(1-\theta) P_{1}+P_{0}+\theta \sum_{\tilde{m}=0}^{1} P_{\tilde{m}}^{H}+P_{2}^{H}\right]\left[V_{K}(m+1)-V_{K}(m)\right] } \\
= & {\left[(1-\theta) P_{1}+P_{0}+\theta \sum_{\tilde{m}=0}^{1} P_{\tilde{m}}^{H}-P_{2}^{H}\right]\left[V_{K}(m)-V_{K}(m-1)\right]+P_{2}^{H} u }
\end{aligned}
$$

Since $V_{K}\left(R_{K}\right)-V_{K}\left(R_{K}-1\right) \leq u, V_{K}\left(R_{K}+1\right)-V_{K}\left(R_{K}\right) \leq u$.

For $m \geq R_{K}+1$,

$$
\begin{aligned}
& {\left[r / \alpha+(1-\theta) P_{1}+P_{0}+\theta \sum_{\tilde{m}=0}^{1} P_{\tilde{m}}^{H}+P_{2}^{H}\right]\left[V_{K}(m+1)-V_{K}(m)\right] } \\
= & {\left[(1-\theta) P_{1}+P_{0}+\theta \sum_{\tilde{m}=0}^{1} P_{\tilde{m}}^{H}\right]\left[V_{K}(m)-V_{K}(m-1)\right] } \\
& +P_{2}^{H}\left[V_{K}(m-1)-V_{K}(m-2)\right]
\end{aligned}
$$

Since $V_{K}\left(R_{K}+1\right)-V_{K}\left(R_{K}\right) \leq u$ and $V_{K}\left(R_{K}\right)-V_{K}\left(R_{K}-1\right) \leq u V_{K}\left(R_{K}+2\right)-V_{K}\left(R_{K}+1\right) \leq$ $u$. Thus, applying the above equation recursively, $V_{K}(m+1)-V_{K}(m) \leq u$ for $m \geq R_{K}+1$.

(b) The uninformed buyers:

Since $\mu_{1}=1, \rho_{K}^{U}(m p, p)=\rho_{K}^{I}(m p)$ for $m \geq 2$.

Thus, the same proof as in (a) is applied to this case.

(c) By the definition of $T_{K}, V_{K}(m)-V_{K}(m-1)>\mu_{0} u$ for $m \leq T_{K}$, and $V_{K}\left(T_{K}+1\right)-$ $V_{K}\left(T_{K}\right) \leq \mu_{0} u$. Thus, it suffices to show: $V_{K}(m)-V_{K}(m-1) \leq \mu_{0} u$ for $m>T_{K}$.

(i) Consider the case that $T_{K}>R_{K}=S_{K}$.

For $m \geq T_{K}+1$, 
By equation (A5),

$$
\begin{aligned}
& {\left[r / \alpha+(1-\theta) P_{1}+P_{0}+\theta \sum_{\tilde{m}=0}^{1} P_{\tilde{m}}^{H}+P_{2}^{H}\right]\left[V_{K}(m+1)-V_{K}(m)\right] } \\
= & {\left[(1-\theta) P_{1}+P_{0}+\theta \sum_{\tilde{m}=0}^{1} P_{\tilde{m}}^{H}\right]\left[V_{K}(m)-V_{K}(m-1)\right] } \\
& +P_{2}^{H}\left[V_{K}(m-1)-V_{K}(m-2)\right]
\end{aligned}
$$

$V_{K}\left(T_{K}+1\right)-V_{K}\left(T_{K}\right) \leq \mu_{0} u$ and $V_{K}\left(T_{K}\right)-V_{K}\left(T_{K}-1\right) \leq u$ implies that if $P_{2}^{H} P_{0}^{L} / P_{0}^{H} \leq$ $r / \alpha$, then $V_{K}\left(T_{K}+2\right)-V_{K}\left(T_{K}+1\right) \leq \mu_{0} u$.

Applying equation (A5) recursively, $V_{K}(m+1)-V_{K}(m) \leq \mu_{0} u$ for $m \geq T_{K}+1$.

(ii) Consider the case that $T_{K} \leq R_{K}=S_{K}$.

For $T_{K}+1 \leq m<R_{K}$ :

Since $V_{K}\left(T_{K}+1\right)-V_{K}\left(T_{K}\right) \leq \mu_{0} u$, by equation $(\mathrm{A} 6) V_{K}(m+1)-V_{K}(m) \leq \mu_{0} u$ for $T_{K}+1 \leq m<R_{K}$.

For $m=R_{K}$ :

By equation (A7), since $V_{K}\left(R_{K}\right)-V_{K}\left(R_{K}-1\right) \leq \mu_{0} u$, if $P_{2}^{H}\left(P_{0}^{L}-P_{0}^{H}\right) / P_{0}^{H} \leq r / \alpha$, $V_{K}\left(R_{K}+1\right)-V_{K}\left(R_{K}\right) \leq \mu_{0} u$.

For $m \geq R_{K}+1$, by equation (A8), since $V_{K}\left(R_{K}+1\right)-V_{K}\left(R_{K}\right) \leq \mu_{0} u$ (if $P_{2}^{H}\left(P_{0}^{L}-P_{0}^{H}\right) / P_{0}^{H} \leq$ $r / \alpha)$ and $V_{K}\left(R_{K}\right)-V_{K}\left(R_{K}-1\right) \leq \mu_{0} u, V_{K}\left(R_{K}+2\right)-V_{K}\left(R_{K}+1\right) \leq \mu_{0} u$. By applying equation (A8) recursively, $V_{K}(m+1)-V_{K}(m) \leq \mu_{0} u$ for $m \geq R_{K}+1$.

LEMMA 14. Suppose that $r / \alpha \geq(1-\theta) P_{0}^{H}$, and if $V_{K}(4)-V_{K}(2)>\gamma$, then $V_{K}(2)-$ $V_{K}(0)>u$.

Then, at the second example of the wealth-signaling equilibrium, it is optimal for buyers

(a) in state up to 2 (in the support) to reject offer $2 p$ or higher,

(b) in state up to $R_{K}$ to reject offer $2 p$,

(c) in state above $R_{K}$ to accept offer $2 p$ made by sellers in state of 2 . 
Proof. (a) Since $V_{K}(2)-V_{K}(0)>u$, the informed and uninformed buyers with 2 units of money reject offer $2 p$.

(b) is shown by $R_{K}=\max \left\{m \mid u+V_{K}(m-2)<V_{K}(m)\right\}$, which implies that for any $n \leq$ $R_{K}, u+V_{K}(n-2)<V_{K}(n)$.

(c) Since $\mu_{2}=1, R_{K}=S_{K}$. It suffices to show $V_{K}(m)-V_{K}(m-2) \leq u$ for $m \geq R_{K}+1$.

(i) Consider the case $R_{K}<T_{K}$.

If $R_{K}+2 \leq T_{K}$, consider the following four cases: (1) $R_{K}+1 \leq m \leq R_{K}+2$, (2) $R_{K}+3 \leq$ $m \leq T_{K}$ (if $R_{K}+2=T_{K},(2)$ is $\left.\emptyset\right),(3) T_{K}+1 \leq m \leq T_{K}+2$, and $(4) T_{K}+3 \leq m$. If $R_{K}+2>T_{K}$, consider the following four cases: (1)' $R_{K}+1 \leq m \leq T_{K}$, (2)' $T_{K}+1 \leq m \leq R_{K}+2$, (3)' $R_{K}+3 \leq m \leq T_{K}+2,(4)^{\prime} T_{K}+3 \leq m$.

Case (1). $V_{K}\left(R_{K}+1\right)-V_{K}\left(R_{K}-1\right) \leq u$ by the definition of $R_{K}$. By equations (9) and (11), for $m=R_{K}+2$,

$$
\begin{aligned}
& {\left[r / \alpha+(1-\theta) P_{1}+\theta \sum_{\tilde{m}=0}^{1} P_{\tilde{m}}^{H}+P_{2}^{H}\right]\left[V_{K}\left(R_{K}+2\right)-V_{K}\left(R_{K}\right)\right] } \\
= & {\left[(1-\theta) P_{1}+\theta \sum_{\tilde{m}=0}^{1} P_{\tilde{m}}^{H}\right]\left[V_{K}\left(R_{K}+1\right)-V_{K}\left(R_{K}-1\right)\right]+P_{2}^{H} u . }
\end{aligned}
$$

Thus, $V_{K}\left(R_{K}+2\right)-V_{K}\left(R_{K}\right) \leq u$. Hence, $V_{K}(m)-V_{K}(m-2) \leq u$ for $R_{K}+1 \leq m \leq R_{K}+2$. Case (2). By equation (11),

$$
\begin{aligned}
& {\left[r / \alpha+(1-\theta) P_{1}+\theta \sum_{\widetilde{m}=0}^{1} P_{\tilde{m}}^{H}+P_{2}^{H}\right]\left[V_{K}(m)-V_{K}(m-2)\right] } \\
= & {\left[(1-\theta) P_{1}+\theta \sum_{\tilde{m}=0}^{1} P_{\tilde{m}}^{H}\right]\left[V_{K}(m-1)-V_{K}(m-3)\right] } \\
& +P_{2}^{H}\left[V_{K}(m-2)-V_{K}(m-4)\right] .
\end{aligned}
$$

Since $V_{K}\left(R_{K}+1\right)-V_{K}\left(R_{K}-1\right) \leq u$ and $V_{K}\left(R_{K}+2\right)-V_{K}\left(R_{K}\right) \leq u$ (by the proof of case (1)), $V_{K}\left(R_{K}+3\right)-V_{K}\left(R_{K}+1\right) \leq u$. It implies $V_{K}(m)-V_{K}(m-2) \leq u$ for $R_{K}+3 \leq m \leq T_{K}$ 
by applying the above equation recursively.

Case (3). By equation (11),

$$
\begin{aligned}
& {\left[r / \alpha+(1-\theta)\left(P_{0}+P_{1}\right)+\theta \sum_{\tilde{m}=0}^{1} P_{\tilde{m}}^{H}+P_{2}^{H}\right]\left[V_{K}(m)-V_{K}(m-2)\right] } \\
= & {\left[(1-\theta)\left(P_{0}+P_{1}\right)+\theta \sum_{\tilde{m}=0}^{1} P_{\tilde{m}}^{H}\right]\left[V_{K}(m-1)-V_{K}(m-3)\right] } \\
& +P_{2}^{H}\left[V_{K}(m-2)-V_{K}(m-4)\right]+(1-\theta) P_{0}^{H} u .
\end{aligned}
$$

Since $V_{K}\left(T_{K}\right)-V_{K}\left(T_{K}-2\right) \leq u$ and $V_{K}\left(T_{K}-1\right)-V_{K}\left(T_{K}-3\right) \leq u$ (by the proof of case (2)),

if $r / \alpha \geq(1-\theta) P_{0}^{H}, V_{K}(m)-V_{K}(m-2) \leq u$ for $T_{K}+1 \leq m \leq T_{K}+2$ by the above equation.

Case (4). By equation (11),

$$
\begin{aligned}
& {\left[r / \alpha+(1-\theta)\left(P_{0}+P_{1}\right)+\theta \sum_{\widetilde{m}=0}^{1} P_{\tilde{m}}^{H}+P_{2}^{H}\right]\left[V_{K}(m)-V_{K}(m-2)\right] } \\
= & {\left[(1-\theta)\left(P_{0}+P_{1}\right)+\theta \sum_{\tilde{m}=0}^{1} P_{\tilde{m}}^{H}\right]\left[V_{K}(m-1)-V_{K}(m-3)\right] } \\
& +P_{2}^{H}\left[V_{K}(m-2)-V_{K}(m-4)\right] .
\end{aligned}
$$

Since $V_{K}\left(T_{K}+1\right)-V_{K}\left(T_{K}-1\right) \leq u$ and $V_{K}\left(T_{K}+2\right)-V_{K}\left(T_{K}\right) \leq u$ if $r / \alpha \geq(1-\theta) P_{0}^{H}$ (by the proof of case (3)), $V_{K}\left(T_{K}+3\right)-V_{K}\left(T_{K}+1\right) \leq u$. It implies $V_{K}(m)-V_{K}(m-2) \leq u$ for $m \geq T_{K}+3$ by applying the above equation recursively.

Case (1)' The proof is the same as the proof of case (1). 
Case (2)' By equations (9) and (11),

$$
\begin{aligned}
& {\left[r / \alpha+(1-\theta)\left(P_{0}+P_{1}\right)+\theta \sum_{\tilde{m}=0}^{1} P_{\tilde{m}}^{H}+P_{2}^{H}\right]\left[V_{K}(m)-V_{K}(m-2)\right] } \\
= & {\left[(1-\theta) P_{1}+\theta \sum_{\tilde{m}=0}^{1} P_{\tilde{m}}^{H}\right]\left[V_{K}(m-1)-V_{K}(m-3)\right] } \\
& +(1-\theta) P_{0}\left[V_{K}(m-1)-V_{K}(m-2)\right]+\left[(1-\theta) P_{0}^{H}+P_{2}^{H}\right] u .
\end{aligned}
$$

Since $V_{K}\left(R_{K}+1\right)-V_{K}\left(R_{K}-1\right) \leq u$ and $V_{K}\left(T_{K}\right)-V_{K}\left(T_{K}-1\right) \leq u$, if $r / \alpha \geq(1-\theta) P_{0}^{H}$, $V_{K}(m)-V_{K}(m-2) \leq u$ for $T_{K} \leq m \leq R_{K}+2$.

Case (3)' The proof is the same as the proof of case (3), except that "(by the proof of case (2))" is replaced with "(by the proof of case (1)')".

Case (4)' The proof is the same as the proof of case (4), except that "(by the proof of case $(3))$ " is replaced with "(by the proof of case (3)')".

(ii) Consider the case $R_{K}>T_{K}$

Furthermore, consider the following two cases: (1)" $R_{K}+1 \leq m \leq R_{K}+2$ and (2)" $R_{K}+3 \leq m$.

Case (1)". $V_{K}\left(R_{K}+1\right)-V_{K}\left(R_{K}-1\right) \leq u$ by the definition of $R_{K}$. By equations (9) and (11), for $m=R_{K}+2$,

$$
\begin{aligned}
& {\left[r / \alpha+(1-\theta)\left(P_{0}+P_{1}\right)+\theta \sum_{\tilde{m}=0}^{1} P_{\tilde{m}}^{H}+P_{2}^{H}\right]\left[V_{K}(m)-V_{K}(m-2)\right] } \\
= & {\left[(1-\theta)\left(P_{0}+P_{1}\right)+\theta \sum_{\tilde{m}=0}^{1} P_{\tilde{m}}^{H}\right]\left[V_{K}(m-1)-V_{K}(m-3)\right]+P_{2}^{H} u . }
\end{aligned}
$$

Thus, $V_{K}(m)-V_{K}(m-2) \leq u$ for $R_{K}+1 \leq m \leq R_{K}+2$. 
Case (2)". By equation (11),

$$
\begin{aligned}
& {\left[r / \alpha+(1-\theta)\left(P_{0}+P_{1}\right)+\theta \sum_{\tilde{m}=0}^{1} P_{\tilde{m}}^{H}+P_{2}^{H}\right]\left[V_{K}(m)-V_{K}(m-2)\right] } \\
= & {\left[(1-\theta)\left(P_{0}+P_{1}\right)+\theta \sum_{\tilde{m}=0}^{1} P_{\tilde{m}}^{H}\right]\left[V_{K}(m-1)-V_{K}(m-3)\right] } \\
& +P_{2}^{H}\left[V_{K}(m-2)-V_{K}(m-4)\right] .
\end{aligned}
$$

Since $V_{K}\left(R_{K}+1\right)-V_{K}\left(R_{K}-1\right) \leq u$ and $V_{K}\left(R_{K}+2\right)-V_{K}\left(R_{K}\right) \leq u$ (by the proof of case $(1)$ "), $V_{K}\left(R_{K}+3\right)-V_{K}\left(R_{K}+1\right) \leq u$. It implies $V_{K}(m)-V_{K}(m-2) \leq u$ for $m \geq R_{K}+3$ by applying the above equation recursively.

(iii) Consider the case $R_{K}=T_{K}$.

Furthermore, consider the following two cases: (1)" $R_{K}+1 \leq m \leq R_{K}+2$ and (2)", $R_{K}+3 \leq m$.

Case (1)"'. $V_{K}\left(R_{K}+1\right)-V_{K}\left(R_{K}-1\right) \leq u$ by the definition of $R_{K}$. By equations $(9)$ and (11), for $m=R_{K}+2$,

$$
\begin{aligned}
& {\left[r / \alpha+(1-\theta)\left(P_{0}+P_{1}\right)+\theta \sum_{\tilde{m}=0}^{1} P_{\tilde{m}}^{H}+P_{2}^{H}\right]\left[V_{K}\left(R_{K}+2\right)-V_{K}\left(R_{K}\right)\right] } \\
= & {\left[(1-\theta) P_{1}+\theta \sum_{\tilde{m}=0}^{1} P_{\tilde{m}}^{H}\right]\left[V_{K}\left(R_{K}+1\right)-V_{K}\left(R_{K}-1\right)\right] } \\
& +(1-\theta) P_{0}\left[V_{K}\left(R_{K}+1\right)-V_{K}\left(R_{K}\right)\right]+\left[(1-\theta) P_{0}^{H}+P_{2}^{H}\right] u .
\end{aligned}
$$

Since $V_{K}\left(R_{K}+1\right)-V_{K}\left(R_{K}-1\right) \leq u$ and $V_{K}\left(R_{K}+1\right)-V_{K}\left(R_{K}\right)=V_{K}\left(T_{K}+1\right)-V_{K}\left(T_{K}\right) \leq$ $\mu_{0} u$, if $r / \alpha \geq(1-\theta) P_{0}^{H}$, then $V_{K}\left(R_{K}+2\right)-V_{K}\left(R_{K}\right) \leq u$.

Case (2)"'. The proof is as the same as the proof of (2)".

Next, find the conditions that offer strategy profile (iii), (iv) and (vi) are optimal. By $A_{K}(\eta)$, (vi) is weakly undominated if and only if $V_{K}\left(M_{K}+Q_{K}\right)-V_{K}\left(M_{K}\right)>\gamma$, i.e., $V_{K}(4)-V_{K}(2)>\gamma$. 
LEMMA 15. Suppose $V_{K}(3)-V_{K}(2) \leq \gamma<V_{K}(2)-V_{K}(1), V_{K}(1)-V_{K}(0)>\gamma$, and if $V_{K}(4)-V_{K}(2)>\gamma$, then $V_{K}(2)-V_{K}(0)>u$.

Then, it is optimal for sellers with money holdings of less than $2 p$ to offer $p$. Furthermore, suppose that if $R_{K}=3$, then $V_{K}(4)-V_{K}(3) \leq \gamma$, then it is optimal for sellers with money holdings of at least $2 p$ to offer a price above $p$.

Proof. (I) Consider a seller with money $m p$ and $m<2$.

By $V_{K}(2)-V_{K}(0)>u$, for all $j>1, R^{I}(j p)+R^{U}(j p, m p)=1$. Since $V_{K}(2)-V_{K}(1)>\gamma$ and $V_{K}(1)-V_{K}(0)>\gamma$, given $R^{I}$ and $R^{U}$, by the net expected value of an agent in state $m$ offering $j p: W(m, j)=\left[1-R^{I}(j p)-R^{U}(j p, m p)\right]\left[V_{K}(m+j)-\gamma-V_{K}(m)\right]$.

For $m=0,1$ and $j>1, W(m, 1)=\left(1-\theta+1_{K} \theta\right) \sum_{\widetilde{m}=1}^{2}\left(P_{\tilde{m}}^{H}+P_{\widetilde{m}}^{L}\right)\left[V_{K}(m+1)-\gamma-V_{K}(m)\right]$ $>W(m, j)=0>W(m, 0)$, since $V_{K}(2)-V_{K}(1)>\gamma$ and $V_{K}(1)-V_{K}(0)>\gamma$. Thus, for $m=0,1$, $\omega(m p)=p$, That is, all agents holding less than $2 p$ units of money should offer exactly $p$.

(II) Next, consider an agent with money $m p$ and $m \geq 2$. To show $\omega(m p)>p$, it is sufficient to show $p \notin A_{K}(\eta)$, or $V_{K}(m+1)-V_{K}(m) \leq \gamma$.

(a) For $m \in\left[2, \min \left(R_{K}, T_{K}\right)\right):$ By lemma showing concavity of $V_{K}$ for $m \leq \min \left(R_{K}, T_{K}\right)$ and $V_{K}(3)-V_{K}(2) \leq \gamma$, for any $m \in\left[2, \min \left(R_{K}, T_{K}\right)\right), V_{K}(m+1)-V_{K}(m) \leq \gamma$.

(i) Consider the case $R_{K}<T_{K}$.

(b) For $m \in\left[R_{K}, T_{K}\right)$ :

(1) Consider the case that $R_{K}>3$.

By the definition of $R_{K}, V_{K}\left(R_{K}+1\right)-V_{K}\left(R_{K}-1\right) \leq u<V_{K}\left(R_{K}\right)-V_{K}\left(R_{K}-2\right)$; thus, $V_{K}\left(R_{K}+1\right)-V_{K}\left(R_{K}\right)<V_{K}\left(R_{K}-1\right)-V_{K}\left(R_{K}-2\right) \leq \gamma$, since $R_{K}-2 \in\left[2, R_{K}\right)$.

For $m \in\left[R_{K}+1, T_{K}\right)$, by equation (11),

$$
\begin{aligned}
& {\left[r / \alpha+(1-\theta) P_{1}+\theta \sum_{\tilde{m}=0}^{1} P_{\tilde{m}}^{H}+P_{2}^{H}\right]\left[V_{K}(m+1)-V_{K}(m)\right] } \\
= & {\left[(1-\theta) P_{1}+\theta \sum_{\tilde{m}=0}^{1} P_{\tilde{m}}^{H}\right]\left[V_{K}(m)-V_{K}(m-1)\right] } \\
& +P_{2}^{H}\left[V_{K}(m-1)-V_{K}(m-2)\right] .
\end{aligned}
$$


Since $V_{K}\left(R_{K}+1\right)-V_{K}\left(R_{K}\right) \leq \gamma$ and $V_{K}\left(R_{K}\right)-V_{K}\left(R_{K}-1\right) \leq \gamma$, starting with $m=R_{K}+1$, and applying equation (A9) recursively, $V_{K}(m+1)-V_{K}(m) \leq \gamma$ for $m \in\left[R_{K}+1, T_{K}\right)$. Hence, $V_{K}(m+1)-V_{K}(m) \leq \gamma$ for $m \in\left[R_{K}, T_{K}\right)$.

(2) Consider the case that $R_{K}=3$. By using the assumption that $V_{K}(4)-V_{K}(3) \leq \gamma$ and $V_{K}(3)-V_{K}(2) \leq \gamma$, and applying equation (A9) recursively, $V_{K}(m+1)-V_{K}(m) \leq \gamma$ for $m \in\left[R_{K}+1, T_{K}\right)$. Hence, $V_{K}(m+1)-V_{K}(m) \leq \gamma$ for $m \in\left[R_{K}, T_{K}\right)$.

(c) For $m=T_{K}:$ By equation (11),

$$
\begin{aligned}
& {\left[r / \alpha+(1-\theta)\left(P_{0}+P_{1}\right)+\theta \sum_{\widetilde{m}=0}^{1} P_{\tilde{m}}^{H}+P_{2}^{H}\right]\left[V_{K}\left(T_{K}+1\right)-V_{K}\left(T_{K}\right)\right] } \\
= & {\left[(1-\theta) P_{1}+\theta \sum_{\widetilde{m}=0}^{1} P_{\tilde{m}}^{H}\right]\left[V_{K}\left(T_{K}\right)-V_{K}\left(T_{K}-1\right)\right] } \\
& +P_{2}^{H}\left[V_{K}\left(T_{K}-1\right)-V_{K}\left(T_{K}-2\right)\right]+(1-\theta) P_{0} \mu_{0} u \\
\leq & {\left[(1-\theta)\left(P_{0}+P_{1}\right)+\theta \sum_{\widetilde{m}=0}^{1} P_{\widetilde{m}}^{H}\right]\left[V_{K}\left(T_{K}\right)-V_{K}\left(T_{K}-1\right)\right] } \\
& +P_{2}^{H}\left[V_{K}\left(T_{K}-1\right)-V_{K}\left(T_{K}-2\right)\right]
\end{aligned}
$$

The last inequality holds since $V_{K}\left(T_{K}\right)-V_{K}\left(T_{K}-1\right)>\mu_{0} u$ by the definition of $T_{K}$. Since $V_{K}\left(T_{K}\right)-V_{K}\left(T_{K}-1\right) \leq \gamma$ and $V_{K}\left(T_{K}-1\right)-V_{K}\left(T_{K}-2\right) \leq \gamma$ by case $(\mathrm{b}), V_{K}\left(T_{K}+1\right)-V_{K}\left(T_{K}\right) \leq$ $\gamma$.

(d) For $m \geq T_{K}+1$ : By equation (11),

$$
\begin{aligned}
& {\left[r / \alpha+(1-\theta)\left(P_{0}+P_{1}\right)+\theta \sum_{\tilde{m}=0}^{1} P_{\tilde{m}}^{H}+P_{2}^{H}\right]\left[V_{K}(m+1)-V_{K}(m)\right] } \\
= & {\left[(1-\theta)\left(P_{0}+P_{1}\right)+\theta \sum_{\tilde{m}=0}^{1} P_{\tilde{m}}^{H}\right]\left[V_{K}(m)-V_{K}(m-1)\right] } \\
& +P_{2}^{H}\left[V_{K}(m-1)-V_{K}(m-2)\right] .
\end{aligned}
$$


Since $V_{K}\left(T_{K}+1\right)-V_{K}\left(T_{K}\right) \leq \gamma$ and $V_{K}\left(T_{K}\right)-V_{K}\left(T_{K}-1\right) \leq \gamma, V_{K}(m+1)-V_{K}(m) \leq \gamma$ for $m \geq T_{K}+1$.

(ii) Consider the case $R_{K} \geq T_{K}$.

(b)' For $m \in\left[T_{K}, R_{K}\right):$ By equation (9),

$$
\begin{aligned}
& {\left[r / \alpha+(1-\theta)\left(P_{0}+P_{1}\right)+\theta \sum_{\tilde{m}=0}^{1} P_{\tilde{m}}^{H}\right]\left[V_{K}(m+1)-V_{K}(m)\right] } \\
= & {\left[(1-\theta)\left(P_{0}+P_{1}\right)+\theta \sum_{\tilde{m}=0}^{1} P_{\tilde{m}}^{H}\right]\left[V_{K}(m)-V_{K}(m-1)\right] }
\end{aligned}
$$

Since $V_{K}\left(T_{K}\right)-V_{K}\left(T_{K}-1\right) \leq \gamma$ by case $(\mathrm{a}), V_{K}(m+1)-V_{K}(m) \leq \gamma$ for $m \in\left[T_{K}, R_{K}\right)$.

(c)' For $m \geq R_{K}: V_{K}\left(R_{K}+1\right)-V_{K}\left(R_{K}\right) \leq \gamma$ by the definition of $R_{K}$. For $m \geq R_{K}+1$, by equation (11),

$$
\begin{aligned}
& {\left[r / \alpha+(1-\theta)\left(P_{0}+P_{1}\right)+\theta \sum_{\tilde{m}=0}^{1} P_{\tilde{m}}^{H}+P_{2}^{H}\right]\left[V_{K}(m+1)-V_{K}(m)\right] } \\
= & {\left[(1-\theta)\left(P_{0}+P_{1}\right)+\theta \sum_{\widetilde{m}=0}^{1} P_{\tilde{m}}^{H}\right]\left[V_{K}(m)-V_{K}(m-1)\right] } \\
& +P_{2}^{H}\left[V_{K}(m-1)-V_{K}(m-2)\right] .
\end{aligned}
$$

Since $V_{K}\left(R_{K}+1\right)-V_{K}\left(R_{K}\right) \leq \gamma$ and $V_{K}\left(R_{K}\right)-V_{K}\left(R_{K}-1\right) \leq \gamma$ by case (b)', $V_{K}(m+1)-$ $V_{K}(m) \leq \gamma$ for $m \geq R_{K}$.

In sum, for all $m \geq 2, V_{K}(m+1)-V_{K}(m) \leq \gamma$.

The discussion and conditions in the first part (before part (I)) and part (II) of Appendix is summarized in Lemma1 (b). 


\section{References}

[1] Berentsen, A., "Money Inventories in Search Equilibrium," Journal of Money, Credit, and Banking 32 (2000), 168-178.

[2] Berentsen, A., "On the Distribution of Money Holdings in a Random-Matching Model," International Economic Review 43 (2002), 945-954.

[3] Berentsen, A., M. Molico, and R. Wright, "Indivisibilities, Lotteries, and Monetary Exchange," Journal of Economic Theory 107 (2002), 70-94.

[4] Camera, G. and D. Corbae, "Money and Price Dispersion," International Economic Review 40 (1999), 985-1008.

[5] Green, E.J. and R. Zhou, "A Rudimentary Random-Matching Model with Divisible Money and Prices," Journal of Economic Theory 81 (1998), 252-271.

[6] Kiyotaki, N. and R. Wright, "On Money as a Medium of Exchange," Journal of Political Economy 97 (1989), 927-954.

[7] Kiyotaki, N. and R. Wright, "A Contribution to the Pure Theory of Money," Journal of Economic Theory 53 (1991), 215-235.

[8] Kiyotaki, N. and R. Wright, "A Search-Theoretic Approach to Monetary Economics," American Economic Review 83 (1993), 63-77.

[9] Molico, M., "The Distribution of Money and Prices in Search Equilibrium," mimeo, Pennsylvania State University, 1998.

[10] Spence, A.M., "Job Market Signaling," Quarterly Journal of Economics 87 (1973), 355-374.

[11] Taber, A., and N. Wallace, "A Matching Model with Bounded Holdings of Indivisible Money," International Economic Review 40 (1999), 961-984. 
[12] Trejos, A. and R. Wright, "Search, Bargaining, Money and Prices," Journal of Political Economy 103 (1995), 118-141.

[13] Williamson, S. and R. Wright, "Barter and Monetary Exchange under Private Information," American Economic Review 84 (1994), 104-123.

[14] Zhou, R., "Individual and Aggregate Real Balances in a Random-Matching Model," International Economic Review 40 (1999), 1009-1038. 


\section{FIGURE 1}

\section{REGION OF THE PARAMETER SPACE FOR EXAMPLE 1 OF THE WEALTH-SIGNALING}

EQUILIBRIUM $(\bar{M}=0.75)$
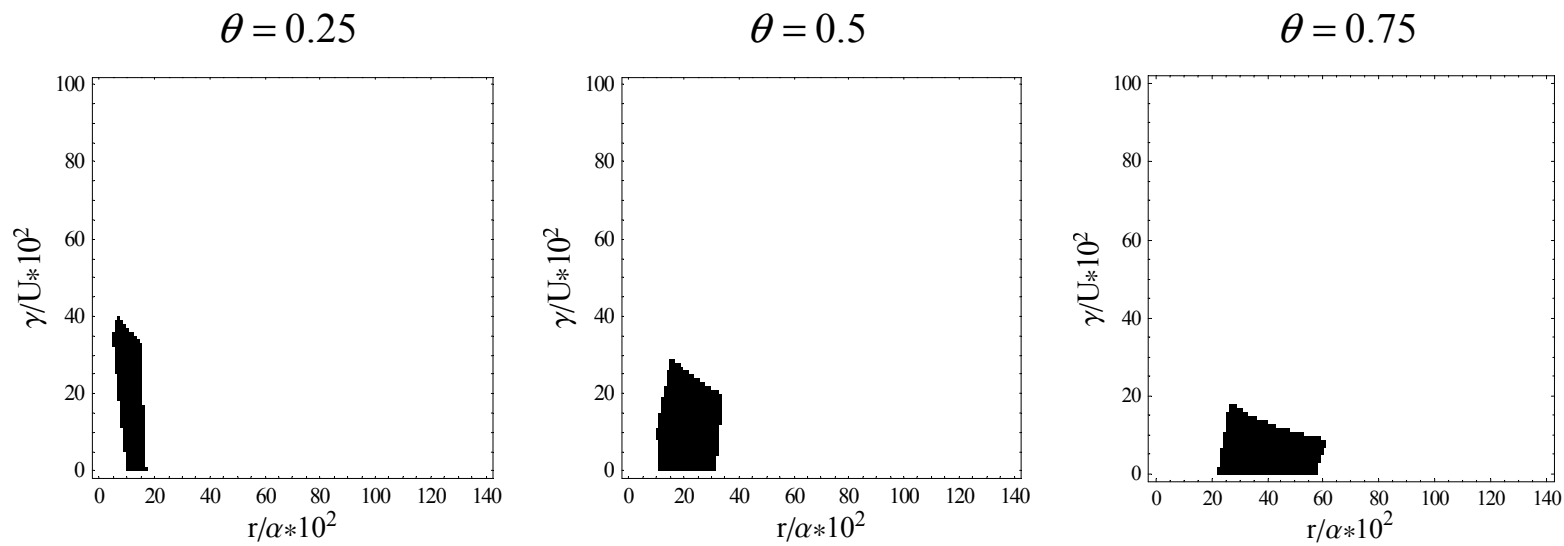


\section{FIGURE 2}

\section{REGION OF THE PARAMETER SPACE FOR EXAMPLE 2 OF THE WEALTH-SIGNALING}

EQUILIBRIUM $(\bar{M}=0.75)$
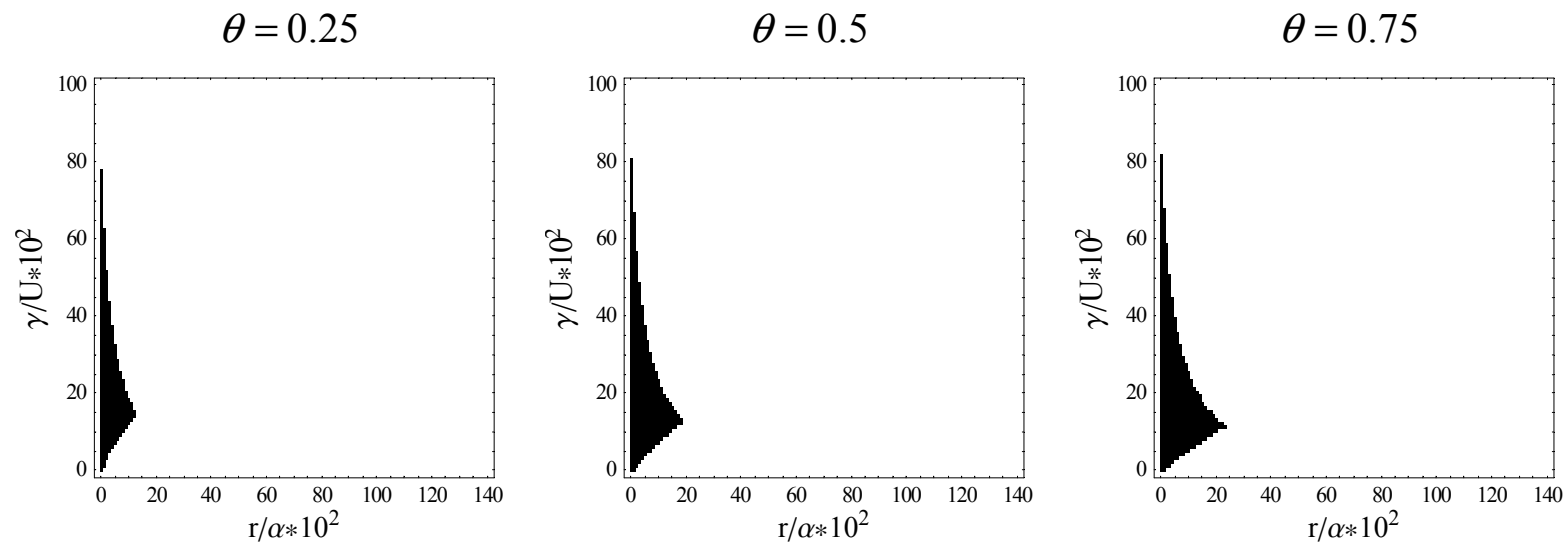
FIGURE 3

REGION OF THE PARAMETER SPACE FOR THE NON-WEALTH-SIGNALING

EQUILIBRIUM $(\bar{M}=0.75)$
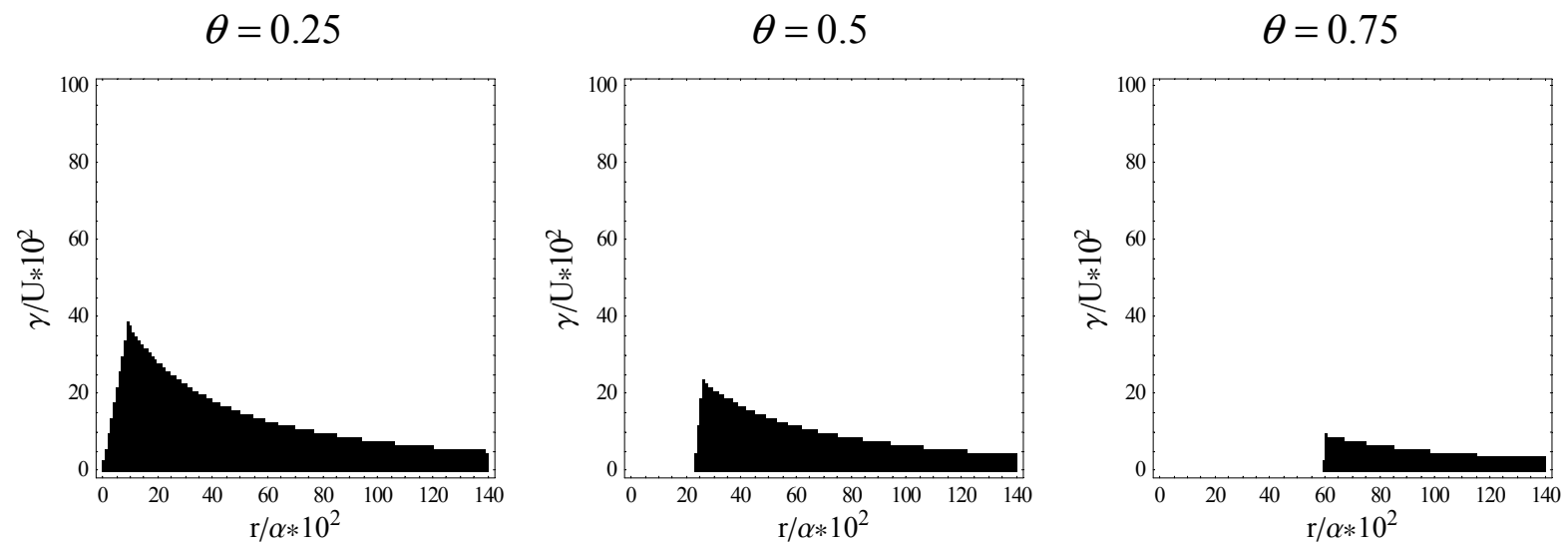


\section{FIGURE 4}

\section{WELFARE FOR HIGH-ABILITY AGENTS $(r / \alpha=0.25)$}
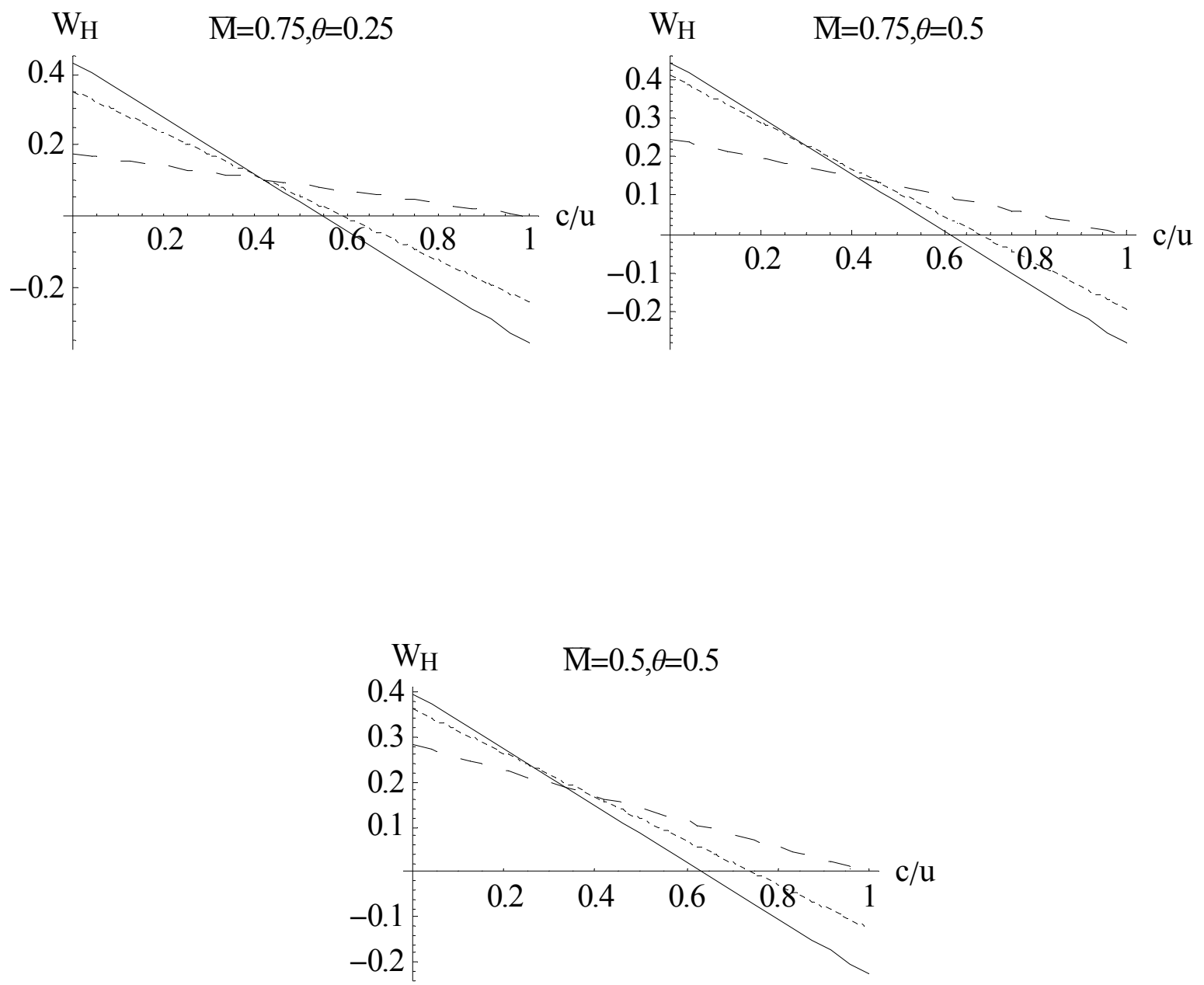

Note: A solid line, a dotted line and a dashed line represent welfare on the non-wealth-signaling equilibrium and on example 1 and example 2 of the wealth-signaling equilibrium respectively. 
FIGURE 5

REGION OF THE PARAMETER SPACE FOR THE FIRST EXAMPLE OF THE WEALTH-SIGNALING EQUILIBRIUM $\quad(\bar{M}=0.1, \theta=0.5)$

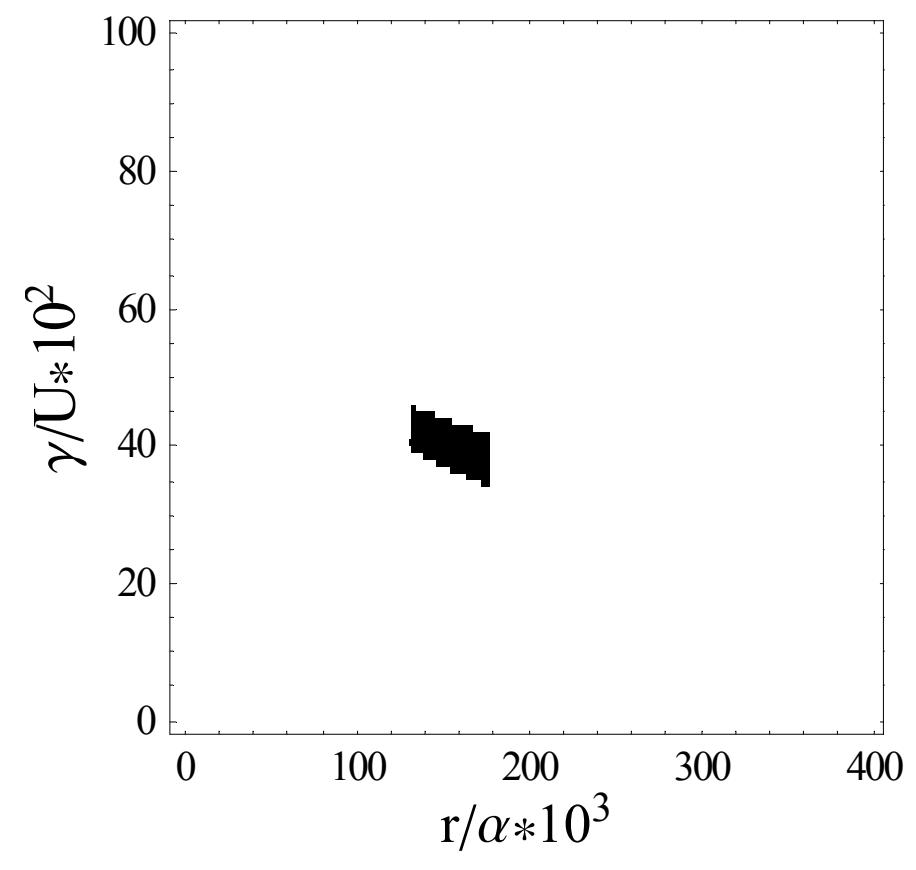


TABLE 1

THE DISTRIBUTION OF WEALTH

EXAMPLE 1 OF THE WEALTH-SIGNALING EQUILIBRIUM $(\bar{M}=0.75)$

\begin{tabular}{rrrrrrrr}
\hline$\theta$ & $P_{0}$ & $P_{1}$ & $P_{2}$ & $\mu_{0}$ & $\mu_{1}$ & $\mu_{2}$ & Gini coef. \\
\hline 0 & 0.46624 & 0.31752 & 0.21624 & 0.5 & 0.5 & 0.5 & 0.557787 \\
0.25 & 0.468417 & 0.31317 & 0.21842 & 0.40657 & 0.553757 & 0.623292 & 0.559618 \\
0.5 & 0.476338 & 0.297323 & 0.226338 & 0.31502 & 0.603792 & 0.752955 & 0.566066 \\
0.75 & 0.495469 & 0.259062 & 0.245469 & 0.21921 & 0.667986 & 0.889474 & 0.580258 \\
1 & 0.558102 & 0.133796 & 0.308102 & 0.104106 & 1 & 1 & 0.613066 \\
\hline
\end{tabular}


TABLE 2

THE DISTRIBUTION OF WEALTH

EXAMPLE 2 OF THE WEALTH-SIGNALING EQUILIBRIUM $(\bar{M}=0.75)$

\begin{tabular}{rrrrrrrr}
\hline$\theta$ & $P_{0}$ & $P_{1}$ & $P_{2}$ & $\mu_{0}$ & $\mu_{1}$ & $\mu_{2}$ & Gini coef. \\
\hline 0 & 0.625 & 0 & 0.375 & 0.2 & & 1 & 0.625 \\
0.25 & 0.583333 & 0.083333 & 0.333333 & 0.142857 & 1 & 1 & 0.62037 \\
0.5 & 0.571429 & 0.107143 & 0.321429 & 0.125 & 1 & 1 & 0.617347 \\
0.75 & 0.563757 & 0.122487 & 0.313757 & 0.113092 & 1 & 1 & 0.614998 \\
1 & 0.558102 & 0.133796 & 0.308102 & 0.104106 & 1 & 1 & 0.613066 \\
\hline
\end{tabular}


TABLE 3

THE DISTRIBUTION OF WEALTH

THE NON-WEALTH-SIGNALING EQUILIBRIUM $(\bar{M}=0.75)$

\begin{tabular}{rrrrrrrr}
\hline$\theta$ & $P_{0}$ & $P_{1}$ & $P_{2}$ & $\mu_{0}$ & $\mu_{1}$ & $\mu_{2}$ & Gini coef. \\
\hline 0 & 0.46624 & 0.31752 & 0.21624 & 0.5 & 0.5 & 0.5 & 0.557787 \\
0.25 & 0.467301 & 0.315397 & 0.217301 & 0.446381 & 0.518087 & 0.589055 & 0.558683 \\
0.5 & 0.472224 & 0.305553 & 0.222224 & 0.374383 & 0.544801 & 0.705335 & 0.562758 \\
0.75 & 0.487808 & 0.274384 & 0.237808 & 0.271173 & 0.598115 & 0.856179 & 0.574809 \\
1 & 0.558102 & 0.133796 & 0.308102 & 0.104106 & 1 & 1 & 0.613066 \\
\hline
\end{tabular}

